\title{
A class of Markov processes with non-linear, bounded generators
}

By

Tadashi UENO

(Received May 23, 1968)

\section{§1. Introduction}

Let $S$ be a locally compact, Hausdorff space, satisfying the second axiom of countability, and let $\boldsymbol{B}(S)$ be the topological Borel field.

Consider a non-linear equation

$$
\begin{gathered}
\frac{\partial u}{\partial t}=u A^{(u)} \\
u=u(t, \cdot) \rightarrow f, \text { as } t \rightarrow 0, *)
\end{gathered}
$$

where the initial value $f$ and the solution $u$ are probability measures on $(S, \boldsymbol{B}(S))$. $u A^{(u)}$ is given by a kernel $A^{(u)}(x, \cdot)$ :

$$
\begin{gathered}
u A^{(u)}(\cdot)=\int_{S} u(d x) A^{(u)}(x, \cdot), \\
(1.2) \quad A^{(u)}(x, \cdot)=\sum_{N=0}^{\infty} \int_{S} \prod_{k=1}^{N} u\left(d x_{k}\right) A_{N}\left(x_{1}, \cdots \cdots, x_{N} \mid x_{2} \cdot\right) \\
A_{N}\left(x_{1}, \cdots \cdots, x_{N} \mid x, E\right) \text { is a measurable function of }\left(x_{1}, \cdots \cdots, x_{N}, x\right)
\end{gathered}
$$

for each $E \in \boldsymbol{B}(S)$, symmetric in $\left(x_{1}, \cdots \cdots, x_{N}\right),{ }^{* *}$ and it is a signed measure in $E$ for each $\left(x_{1}, \cdots \cdots, x_{N}, x\right)$ such that

satisfies

$$
C_{N}=\left\|A_{N}\right\|=\sup _{\left(x_{1}, \ldots, x_{N}, x\right)} \text { total variation of } A_{N}\left(x_{1}, \cdots \cdots, x_{N} \mid x, \cdot\right)
$$

$$
\sum_{N=0}^{\infty} C_{N} N^{P} \leqslant L^{P} \cdot P !, P=1,2, \cdots \cdots
$$

Moreover, $A_{N}$ is a stochastic generator kernel with parameter $\left(x_{1}, \cdots \cdots, x_{N}\right)$, that is,

$$
A_{N}\left(x_{1}, \cdots \cdots, x_{N} \mid x, E\right)=q_{N}\left(x_{1}, \cdots \cdots, x_{N} \mid x\right)\left(\pi_{N}\left(x_{1}, \cdots \cdots, x_{N} \mid x, E\right)-\delta_{\{x\}}(E)\right),
$$

where $\pi_{N}$ is a probability measure in $E$ with mass 0 on $\{x\}$, and $q_{N}$ is non-negative. In a word, $A^{(u)}$ is a stochastic generator kernel bounded by (1.3) and depends multilinearly on $u$ in the sense of (1.2).

We solve equation (1.1) by constructing a fundamental solution $P^{f}(t, x, E)$, which satisfies

$$
\begin{gathered}
\frac{\partial}{\partial t} P^{f}(t, x, \cdot)=\int_{S} P^{f}(t, x, d y) A^{f t}(y, \cdot) \\
f_{t}(\cdot)=\int_{S} f(d x) P^{f}(t, x, \cdot \cdot) .
\end{gathered}
$$

More precisely, we have

THEOREM 1. For a probability measure $f$ on $(S, \boldsymbol{B}(S))$ and $t>0$, there is a

*) $u(t, \cdot) \rightarrow f(\cdot)$ means $u(t, E) \rightarrow f(E)$ and $\frac{\partial u}{\partial t}(E)$ is $\lim _{\Delta \rightarrow 0} \frac{1}{\Delta}(u(t+\Delta, E)-u(t, E)$ ) for each $E$ in $\boldsymbol{B}(S)$.

**) Symmetry is not necessary. Any $A_{N}$ can be replaced by a symmetric kernel $(N !)^{-1} \sum A_{N}\left(x_{\sigma(1)}, \ldots, \chi_{\sigma(N)} \mid x,\right)$, where $\sigma$ ranges over all permutations of $\{1,2, \cdots \cdots, N\}$. 
probability measure $P^{f}(t, x, E)$, which is measurable in $x$ for each $E \in \boldsymbol{B}(S)$, and satisfies

$$
\begin{gathered}
P^{f}(s+t, x, \cdot)=\int_{S} P^{f}(s, x, d y) P^{f s}(t, y, \cdot) \\
f_{s}(\cdot)=\int_{S} f(d x) P^{f}(s, x, \cdot) \\
\lim _{t \rightarrow 0} \frac{1}{t}\left(\int_{S} P^{f}(t, x, d y) \varphi(y)-\varphi(x)\right)=A^{f} \varphi(x)=\int_{S} A^{f}(x, d y) \varphi(y)
\end{gathered}
$$

for any bounded measurable function $\varphi$ on $S$. Here, the convergence is uniform in $x \in S$. Moreover,

$$
\begin{aligned}
& \frac{\partial}{\partial t} \int_{S} P^{f}(t, x, d y) \varphi(y)=\int_{S} P^{f}(t, x, d y) A^{f t} \varphi(y) \\
& \int_{S} P^{f}(t, x, d y) \varphi(y) \text { is analytic in } t \geq 0 .
\end{aligned}
$$

Thus, it is clear that $u(t, \cdot)=f_{t}(\cdot)$ satisfies $(1.1)$.

We now define, by (1.4), a temporally inhomogeneous Markov process $M=(W=$ $\left.S(0, \infty), P^{f}, x(t, w)\right)$ with state space $S$, initial distribution $f$ and transition probability

$$
\begin{aligned}
& P(s, x, t, E)=P^{f s}(t-s, x, E)^{*)} \\
& f_{s}(\cdot)=\int_{S} f(d x) P^{f}(s, x, \cdot) .
\end{aligned}
$$

Attached to this Markov process, we define a series of n-dimensional temporally homogeneous Markov processes $M_{n}=\left(W_{n}=\left(S^{n}\right)[0, \infty), P_{n}^{f}, x_{n}(t, w)\right)$ with state space $S^{n}$, initial distribution $f^{(n)}$, the $n$-fold direct product measure of $f$, and with generator $G_{n}$, where

$$
\begin{gathered}
G_{n} \varphi\left(x_{1}, \cdots \cdots, x_{n}\right)=\sum_{N=0}^{n-1} n^{-N} \sum_{\left(j_{1}, \ldots, j_{N}, i\right)}^{(n)} \int_{S} A_{N}\left(x_{j_{1}}, \cdots \cdots, x_{j_{N}}\right. \\
\left.\mid x_{i}, d y\right) \varphi(x_{1}, \cdots \cdots, \overbrace{y}^{i}, \cdots \cdots, x_{n})
\end{gathered}
$$

for $\varphi$ in $B\left(S^{n}\right)$ which is the space of all bounded measurable functions on $S^{n}$. Here $\sum_{\left(j_{1}, \ldots, j_{N}, i\right)}^{(n)}$ is the sum over $j_{1}, \cdots \cdots, j_{N}, i$ ranging over $1, \cdots \cdots, n$, except that no value is taken at the same time. This is possible, because $G_{n}$ is a bounded operator, and $e^{t G_{n}}$ defines a non-negative, strongly continuous semigroup on $B\left(S^{n}\right)$, and $e^{t G_{n}} 1$ $=1$. Then, it is clear that the coordinate process $x_{n}^{(i)}(t, w)$ of $x_{n}(t)=\left(x_{n}^{(1)}(t), \cdots \cdots, x_{n}^{(n)}(t)\right)$ are not mutually independent, except at time $\left.t=0 .^{* *}\right)$ But, on the other hand, we have the following result.

THEOREM 2. For $0<t_{1}<\cdots \cdots<t_{k}<1 / 2 L\left(3+C_{0}\right)$

$$
\begin{aligned}
& \lim _{n \rightarrow \infty} P_{n}^{f}\left(x_{n}^{(1)}\left(t_{1}\right) \in A_{1}, \cdots \cdots, x_{n}^{(1)}\left(t_{k}\right) \in A_{k}, x_{n}^{(2)}\left(t_{1}\right) \in B_{1}, \cdots \cdots, x_{n}^{(2)}\left(t_{k}\right) \in B_{k}\right) \\
(1.10) & =P^{f}\left(x\left(t_{1}\right) \in A_{1}, \cdots \cdots, x\left(t_{k}\right) \in A_{k}\right) \cdot P^{f}\left(x\left(t_{1}\right) \in B_{1}, \cdots \cdots, x\left(t_{k}\right) \in B_{k c}\right) \\
& =\lim _{n \rightarrow \infty} P_{n}^{f}\left(x_{n}^{(1)}\left(t_{1}\right) \in A_{1}, \cdots \cdots, x_{n}^{(1)}\left(t_{k}\right) \in A_{k}\right) \lim _{n \rightarrow \infty} P_{n}^{f}\left(x_{n}^{(2)}\left(t_{1}\right) \in B, \cdots \cdots, x_{n}^{(2)}\left(t_{k}\right) \in B_{k}\right)
\end{aligned}
$$

*) The proof of Chapman-Kolmogorov equation for $P(s, x, t, \cdot)$ is easy by (1.4). This model shows that for temporally inhomogeneous Markov processes it is not always natural to discuss transition probability and initial distribution separately, while this has been rather customary.

**) We neglect the trivial case, where $A_{N}\left(x_{1}, \cdots \cdots, x_{n} \mid x, \cdot\right) \equiv 0$ for all $N \geq 1$. 
Here, the number of coordinate processes of $x_{n}(t)$ can be replaced by any fixed number.

Considered as motions of $n$ different particles on the space $S$, the coordinate processes $x_{n}^{(1)}(t), \cdots \cdots, x_{n}^{(n)}(t)$ of $x_{n}(t)$ have mutual interactions. But, Theorem 2 shows that these motions get gradually independent as the number of particles increases, and in the limit, they are mutually independent, and each of these particles moves by the law determined by the equation (1.1). This is the so-called propagation of chaos discovered by Mark Kac [3]. Moreover, the $N$ th term $\int_{S} \prod_{k=1}^{N} f\left(d x_{k}\right) A_{N}\left(x_{1}, \cdots \cdots, x_{N} \mid x, \cdot\right)$ of kernel $A^{(f)}$ can be considered as the influence of other $N$ particles on the motion of one particle under observation. So, if the interactions among $N+1$ particles do not actually exist, then $A_{N}=0$. For instance, in the case of the one dimensional model of Boltzmann equation (5.1) for Maxwellian gas by Kac, the kernel $A^{f}$ is given by

$$
\begin{aligned}
& A^{f}(x, d y)=\int_{R 1} f\left(d x_{1}\right) A_{1}\left(x_{1} \mid x, d y\right) \\
& \begin{array}{l}
A_{1}\left(x_{1} \mid x, d y\right)=\frac{1}{\pi} \cdot \frac{1}{\sqrt{x^{2}+x_{1}^{2}-y^{2}}} d y-\delta_{x}(d y), \text { if } x^{2}+x_{1}{ }^{2}>y^{2} \\
=0, \quad \text { if otherwise, }
\end{array}
\end{aligned}
$$

where the effective interactions are limited to binary collisions alone.

Now it is clear that the model we have discussed in this section is a special case of the class of Markov processes with non-linear generators, the field on which Henry McKean [4] made a first step of systematic study. I also note here that this article is a natural extension of Dudley Johnson's works $[1,2]$, where he made a complete description of 2-state space case. I wish to express my thanks to Henry McKean for his interesting talks and discussions on the field, and to Dudley Johnson who sent me his manuscript before publication, and also to Keniti Sato who read my manuscript very carefully and kindly suggested me necessary corrections.

\section{§2. Derivation $D$ and $e^{t D}$}

Let I be an infinite set of indices and let $S^{I}$ be the direct product $\prod_{j \in I} S_{j}$ of copies $S_{j}$ of $S$ with the product topology induced by $S$. We consider real valued bounded functions on $S^{I}$, measurable with respect to the topological Borel field $\boldsymbol{B}\left(S^{I}\right)$ of $S^{I}$ and consider the norm

$$
\|\varphi\|=\sup _{\xi \in S^{I}}|\varphi(\xi)|
$$

We say that $\sum_{j=1}^{\infty} \varphi_{j}$ is convergent in norm, if $\sum_{j=1}^{\infty}\left\|\varphi_{j}\right\|<\infty . \quad F_{0}$ is the set of all functions which depend only on finite variables and $F$ is the set of all function $\varphi$ which can be written as

$$
\varphi=\sum_{j=1}^{\infty} \varphi_{j}, \quad \varphi_{j} \in F_{0},
$$

where the convergence is in norm.

For a subset $J$ of $I$ and functions $\varphi$ and $\phi$ in $F$, we write 


$$
\varphi \equiv \psi \quad(\bmod J)
$$

and say that $\varphi$ and $\phi$ are equivalent modulo $J$, if there are expansions

$$
\varphi=\sum_{j=1}^{\infty} \varphi_{j}, \phi=\sum_{j=1}^{\infty} \psi_{j}, \quad \varphi_{j}, \phi_{j} \in F_{0},
$$

converging in norm, and there is a class of one to one mappings $T_{j}$ from $J$ onto $J$ such that $\phi_{j}$ coincides with the function obtained from $\varphi_{j}$ by the change of variables induced by $T_{j}$. Then, the following are clear by definition.

$$
\begin{aligned}
& \varphi_{1} \equiv \phi_{1}, \varphi_{2} \equiv \phi_{2}^{\prime} \quad(\bmod J) \text { imply } \\
& \varphi_{1}+\varphi_{2} \equiv \phi_{1}+\phi_{2} \quad(\bmod J) . \\
& \varphi \equiv \phi(\bmod J), \text { and } I\left(\varphi^{\prime}\right) \subset J^{c} \text { imply*) } \\
& \varphi \cdot \varphi^{\prime} \equiv \phi \cdot \varphi^{\prime} \quad(\bmod J),
\end{aligned}
$$

where $I(\varphi)$ is the set of indices on which $\varphi$ actually depends.

$$
\begin{aligned}
& \varphi \equiv \phi(\bmod J), \text { and } J \subset J^{\prime} \text { imply } \\
& \varphi \equiv \phi\left(\bmod J^{\prime}\right) .
\end{aligned}
$$

For a probability measure $f$ on $(S, \boldsymbol{B}(S))$, subset $J$ of $I$ and $\varphi \in F$, we write

$$
\int_{J} f^{\infty} \varphi=\int_{S^{J}} \prod_{i \in J} f\left(d x_{i}\right) \varphi(\xi)
$$

where the integration is limited to variables with indices in $J$ and hence the integral is a function of variables with indices in $J^{c}$. It is clear by definition that

$$
\begin{gathered}
\varphi \equiv \phi \quad(\bmod J), \text { and } J \subset J^{\prime} \text { imply } \\
\int_{J^{\prime}} f^{\infty} \varphi=\int_{J^{\prime}} f^{\infty} \psi
\end{gathered}
$$

If $\varphi=\sum_{j=1}^{\infty} \varphi_{j}, \varphi_{j} \in F_{0}$, is convergent in norm,

$$
\int_{J} f^{\infty} \varphi=\sum_{j=1}^{\infty} \int_{J \cap I(\varphi j)} f^{\infty} \varphi_{j}=\sum_{j=1}^{\infty} \int_{S J \cap I(\varphi j)} \prod_{i \in J \cap I\left(\varphi_{j}\right)} f\left(d x_{i}\right) \varphi_{j}
$$

holds by the bounded convergence theorem. In this expression we have to consider only the finite product measure of $f$.

Take a finite subset $I_{0}$ of $I$. We define for $\varphi \in F_{0}$,

$$
Q(N) \varphi=\sum_{i \in I(\varphi)} A_{N}^{(i)} \varphi\left(x_{i_{1}}, x_{i_{2}}, \cdots \cdots, x_{i_{N}} \mid x_{i}\right) .
$$

Here, $A_{N}^{(i)} \varphi$ is the function obtained by applying $A_{N}$ to $\varphi$ as a function of $x_{i}$, keeping other variables fixed. $i_{1}, i_{2}, \cdots \cdots, i_{N}$ are $N$ distinct indices which are not contained in $I(\varphi) \cup I_{0} . Q(N) \varphi$, having variables with indices in $I(\varphi)$ and $i_{1}, \cdots \cdots, i_{N}$, depends on the choice of $i_{1}, \cdots \cdots, i_{N}$. But, it is determined uniquely modulo $I_{0}{ }^{c}$, independent from the choice of $i_{1}, i_{2}, \cdots \cdots, i_{N}$. We simply write $Q(N) \varphi$ for an arbitrary representative of the equivalence class.

We define $Q(N) \varphi$ for $\varphi \in F$ by

$$
Q(N) \varphi=\sum_{j=1}^{\infty} Q(N) \varphi_{j}
$$

*) $J^{c}$ denotes the complement of $J$, that is, $I-J$. 
if there is a convergent series $\sum_{j=1}^{\infty} \varphi_{j}=\varphi, \varphi_{j} \in F_{0}$, such that the series $\sum_{j=1}^{\infty} \sum_{i \in I(\varphi)} A_{N}^{(i)} \varphi_{j}$ $\left(x_{i_{1}}, \cdots \cdots, x_{i_{N}} \mid x_{i}\right)$ is convergent in norm. $Q(N) \varphi$, if exists, is determined uniquely modulo $I_{0}{ }^{c}$, independent of the expression $\varphi=\sum_{j=1}^{\infty} \varphi_{j}$. In fact, by the assumption on the convergence, $Q(N) \varphi$ coincides with $\sum_{i \in I(\varphi)}^{\infty} A_{N}^{(i)} \varphi\left(x_{i_{1}}, \cdots \cdots, x_{i_{N}} \mid x_{i}\right)$ which is convergent and clearly independent on $\left\{\varphi_{j}\right\} . D \varphi$ is defined for $\varphi \in F$ by

$$
D \varphi=\sum_{N=0}^{\infty} Q_{N}(\varphi),
$$

if there is an expression $\varphi=\sum_{i=0}^{\infty} \varphi_{i}, \varphi_{j} \in F_{0}$ such that

$$
\sum_{j=1}^{\infty} \sum_{N=0}^{\infty} \sum_{i \in I\left(\varphi_{j}\right)} A_{N}^{(i)} \varphi_{j}\left(x_{i_{1}},{ }_{N}, \cdots \cdots, x_{i_{N},{ }_{N}} \mid x_{i}\right)
$$

is convergent in norm. $D^{p} \varphi$ and $e^{t D} \varphi$ are defined for $\varphi \in F$ similarly by

$$
D^{p} \varphi=D\left(D^{p-1} \varphi\right), \quad D^{1} \varphi=D \varphi, \quad e^{t D} \varphi=\sum_{n=0}^{\infty} \frac{t^{n}}{n !} D^{n} \varphi,
$$

if there is an expression $\sum_{j=1}^{\infty} \varphi_{j}$ such that the corresponding series in terms of $A_{N}^{(i)}$ are convergent in norm for new indices.

These quantities clearly depend on the choice of variables, but are determined modulo $I_{0}{ }^{C}$. It is clear that $D^{p} \varphi$ and $e^{t D} \varphi$ again belong to $F$ when they exist. Note that variables are added entirely anew for each application of $Q(N)$, and may possibly differ for different $\varphi_{j}$ 's in $\sum_{j=1}^{\infty} \varphi_{j}$ even for a same $Q(N)$. Here, we always assume that $I$ is sufficiently large so that we can pick up new indices freely from $I$.

When $I(\phi)$ is contained in $I_{0}$, then

$$
\phi \cdot Q(N) \varphi, \quad \phi \cdot D^{p} \varphi, \quad \psi \cdot e^{t D} \varphi
$$

are determined modulo $I_{0}{ }^{c}$, by virtue of (2.2). Similarly, if all variables of $\varphi_{1}, \varphi_{2}$, $\cdots \cdots, \varphi_{n}$ have indices contained in $I_{0}$, then

$$
Q\left(N_{1}\right) \varphi_{1} \cdots \cdots Q\left(N_{n}\right) \varphi_{n}, \quad D^{p_{1}} \varphi_{1} \cdots \cdots D^{p_{n}} \varphi_{n}, \quad e^{t_{1} D} \varphi_{1} \cdots \cdots e^{t_{n} D} \varphi_{n}
$$

are all determined modulo $I_{0}{ }^{c}$. Here, we write, for instance,

for abbreviation.

$$
D^{p_{1}} \varphi_{1} \cdots \cdots D^{p_{n}} \varphi_{n} \quad \text { for } D^{p_{1}}\left(\varphi_{1} \cdot D^{p_{2}}\left(\varphi_{2} \cdot \cdots \cdots D^{p_{n-1}}\left(\varphi_{n-1} \cdot D^{p_{n}} \varphi_{n}\right)\right) \cdots \cdots\right)
$$

Now, following are the fundamental properties of $e^{t D}$.

LEMMA 1. If $\varphi$ and $\phi \in F$ have no indices in common, there are those representatives of $e^{t D} \varphi$ and $e^{t D} \phi$ which have no indices in common. Then, $e^{t D}(\varphi \cdot \phi)$ is equivalent to the product of such representatives modulo $I_{0}{ }^{c}$. This fact is written symbolically by

$$
e^{t D}(\varphi \otimes \phi) \equiv e^{t D} \varphi \otimes e^{t D} \psi \quad\left(\bmod I_{0}^{c}\right) .
$$

For $\varphi \in F$, we have

$$
e^{(s+t) D} \varphi \equiv e^{s D} e^{t D} \varphi .
$$

$\left(\bmod I_{0}{ }^{c}\right)$

Here, the existence of the above quantities are assumed. 
Note that (2.6) depends on the choice of representatives on the right.

COROLLARY. By the notation of Lemma 1, we have

(2.8) $\quad e^{t D}\left(\varphi_{1} \otimes \cdots \cdots \otimes \varphi_{n}\right) \equiv e^{t D} \varphi_{1} \otimes \cdots \cdots \otimes e^{t D} \varphi_{n} \quad\left(\bmod I_{0}{ }^{c}\right)$.

If $\varphi_{1}, \cdots \cdots, \varphi_{n}$ and $\phi_{1}, \cdots \cdots, \phi_{n}$ have variables with indices in $I_{0}$ and

$$
\begin{aligned}
& I\left(\varphi_{1}\right) \cup \cdots \cdots \cup I\left(\varphi_{n}\right) \text { and } I\left(\phi_{1}\right) \cup \cdots \cdots \cup I\left(\phi_{n}\right) \text { are disjoint, then, } \\
& e^{t_{1} D}\left(\varphi_{1} \otimes \phi_{1}\right) e^{t_{2} D}\left(\varphi_{2} \otimes \phi_{2}\right) \cdots \cdots e^{t_{n} D}\left(\varphi_{n} \otimes \phi_{n}\right) \\
& \quad \equiv\left(e^{t_{1} D} \varphi_{1} e^{t_{2} D} \varphi_{2} \cdots \cdots e^{t_{n} D} \varphi_{n}\right) \otimes\left(e^{t_{1} D} \phi_{1} \cdots \cdots e^{t_{n} D} \psi_{n}\right) \quad\left(\bmod I_{0}^{c}\right) \text {, }
\end{aligned}
$$

where $\varphi_{1}, \cdots \cdots, \varphi_{n}$ may have indices in common, and so may $\phi_{1}, \cdots \cdots, \phi_{n}$. More precisely, there are those representatives of the right hand factors that have no indices in common, and the left hand side is equivalent to the product of those representatives. Here, the quantities above are assumed to exist.

Proof of Lemma 1. By the definition of $A_{N}^{(i)}$ and $Q(N)$, we have

$$
\begin{aligned}
& Q(N)(\varphi \otimes \psi)=\sum_{i \in I(\varphi \otimes \psi)} A_{N}^{(i)}(\varphi \otimes \psi) \\
& =\sum_{i \in I(\varphi)+I(\psi)} A_{N}^{(i)}(\varphi \otimes \psi)=\sum_{i \in I(\varphi)} A_{N}^{(i)}(\varphi \otimes \psi)+\sum_{i \in I(\psi)} A_{N}^{(i)}(\varphi \otimes \psi) \\
& =\sum_{i \in I(\varphi)} \phi \otimes A_{N}^{(i)} \varphi+\sum_{i \in I(\psi)} \varphi \otimes A_{N}^{(i)} \psi \\
& =\psi \otimes Q(N \varphi)+\varphi \otimes Q(N) \psi, *)
\end{aligned}
$$

for any possible choice of $i, \cdots \cdots, i_{N}$. This implies

$$
D(\varphi \otimes \psi) \equiv \varphi \otimes D \phi+\phi \otimes D \varphi\left(\bmod I_{0}^{c}\right),
$$

if the indices of $\varphi$ and $\phi$ are avoided for $D \phi$ and $D \varphi$, respectively.

Choosing indices so that new indices are added never in common for $D^{p} \varphi$ and $D^{q} \psi$, and repeating the same procedure, we can extend this relation to

$$
D^{n}(\varphi \otimes \psi) \equiv \sum_{p=0}^{n}\left(\begin{array}{l}
n \\
p
\end{array}\right) D^{n-p} \varphi \otimes D^{p} \psi\left(\bmod I_{0}^{c}\right) .
$$

This naturally implies (2.6) under the condition that representatives of $e^{t D} \varphi$ and $e^{t D} \phi$ have no indices in common.

Since both sides of (2.7) are determined modulo $I_{0}{ }^{c}$ independent on the choice of indices, it is sufficient to find those representatives which are equivalent modulo $I_{0}{ }^{c}$. But, this is clear by formal expansions:

$$
\begin{aligned}
e^{(s+t) D} \varphi & =\sum_{n=0}^{\infty} \frac{1}{n !}(s+t)^{n} D^{n} \varphi . \\
& =\sum_{n=0}^{\infty} \frac{1}{n !} \sum_{p=0}^{n}\left(\begin{array}{l}
n \\
p
\end{array}\right) s^{n-p} t^{p} D^{n-p} D^{p} \varphi . \\
e^{s D}\left(e^{t D} \varphi\right) & =e^{s D}\left(\sum_{p=0}^{\infty} \frac{t^{p}}{p !} D^{p} \varphi\right) \\
& =\sum_{q=0}^{\infty} \sum_{p=0}^{\infty} \frac{1}{q ! p !} s^{q} t^{p} D^{q} D^{p} \varphi=\sum_{n=0}^{\infty} \sum_{p+q=n}^{\infty} \frac{1}{p ! q !} s^{q} t^{p} D^{q} D^{p} \varphi
\end{aligned}
$$

*) We denote by $\varphi \otimes \psi$ the product of $\varphi$ and $\psi$ which have no indices in common. By $A+B$, we mean $A \cup B$ when $A \cap B=\phi$. 


$$
=\sum_{n=0}^{\infty} \frac{1}{n !} \sum_{p=0}^{n}\left(\begin{array}{l}
n \\
p
\end{array}\right) s^{n-p} t^{p} D^{n-p} D^{p} \varphi
$$

Proof of the Corollary. (2.8) is obtained by a simple induction.

$$
\begin{gathered}
e^{t D}\left(\varphi_{1} \otimes \cdots \cdots \otimes \varphi_{n}\right) \equiv e^{t D}\left(\left(\varphi_{1} \otimes \cdots \cdots \otimes \varphi_{n-1}\right) \otimes \varphi_{n}\right) \\
\quad=e^{t D}\left(\varphi_{1} \otimes \cdots \cdots \otimes \varphi_{n-1}\right) \otimes e^{t D} \varphi_{n} \quad\left(\bmod I_{0}^{c}\right) .
\end{gathered}
$$

(2.9) is true for $n=1$ by (2.5). Assume that this is true for $n-1$, and let $\varphi_{0}$ and $\psi_{0}$ be such representatives of $e^{t_{2} D} \varphi_{2} \cdots \cdots e^{t_{n} D} \varphi_{n}$ and $e^{t_{2} D} \phi_{2} \cdots \cdots e^{t_{n} D} \psi_{n}$ in the corollary, respectively. Since there are no indices in common for $\varphi_{0}$ and $\phi_{1}$, and also for $\phi_{0}$ and $\varphi_{1}$, the left hand side of (2.9) is equivalent to

$$
\begin{aligned}
& e^{t_{1} D}\left(\left(\varphi_{1} \otimes \psi_{1}\right) \cdot\left(\varphi_{0} \otimes \psi_{0}\right)\right) \equiv e^{t_{1} D}\left(\left(\varphi_{1} \cdot \varphi_{0}\right) \otimes\left(\psi_{1} \cdot \phi_{0}\right)\right) \\
& \equiv e^{t_{1} D}\left(\varphi_{1} \cdot \varphi_{0}\right) \otimes e^{t_{1} D}\left(\phi_{1} \cdot \psi_{0}\right) \quad\left(\bmod I_{0}^{c}\right),
\end{aligned}
$$

which completes the proof.

LEMma 2. If $I\left(\varphi_{1}\right) \cup \cdots \cdots \cup \cup\left(\varphi_{n}\right)$ is a set of $M$ indices contained in $I_{0}$, then the following estimates hold, independently on the possible choice of new indices.

$$
\begin{aligned}
& \left\|D^{p_{1}} \varphi_{1} \cdots \cdots D^{p_{n}} \varphi_{n}\right\| \leq 2\left(p_{1}+\cdots \cdots+p_{n}+1\right) !(2(M+1) L)^{p_{1}+\cdots+p_{n}}\left\|\varphi_{1}\right\| \cdots \cdots\left\|\varphi_{n}\right\| \\
& \left\|e^{t_{1} D} \varphi_{1} \cdots \cdots e^{t_{n} D} \varphi_{n}\right\| \leq\left(1-2 L(M+1)\left(t_{1}+\cdots \cdots+t_{n}\right)\right)^{-2}\left\|\varphi_{1}\right\| \cdots \cdots\left\|\varphi_{n}\right\| \\
& \text { for } t_{1}+\cdots \cdots+t_{n}<(2 L(M+1))^{-1} \text {. }
\end{aligned}
$$

Proof. Taking care of the number of variables, we have

$$
\begin{aligned}
& \left\|Q(N) \varphi_{1}\right\| \leq \sum_{i \in I\left(\varphi_{1}\right)}\left\|A_{N}^{(i)} \varphi_{1}\right\| \leq \sum_{i \in I\left(\varphi_{1}\right)}\left\|A_{N}\right\| \cdot\left\|\varphi_{1}\right\| \leq M \cdot C_{N} \cdot\left\|\varphi_{1}\right\|, \\
& \left\|Q\left(N_{n}\right) \varphi_{n} \cdots \cdots Q\left(N_{1}\right) \varphi_{1}\right\| \leq\left(M+N_{1}+\cdots \cdots+N_{n-1}\right) C_{N_{n}}\left\|\varphi_{n} \cdot Q\left(N_{n-1}\right) \varphi_{n-1} \cdots \cdots Q\left(N_{1}\right) \varphi_{1}\right\| \\
& \quad \leq\left(M+N_{1}+\cdots \cdots+N_{n-1}\right) C_{N_{n}}\left\|\varphi_{n}\right\| \cdot\left\|Q\left(N_{n-1}\right) \varphi_{n-1} \cdots \cdots Q\left(N_{1}\right) \varphi_{1}\right\| \\
& \quad \leq M\left(M+N_{1}\right) \cdots \cdots\left(M+N_{1}+\cdots \cdots+N_{n-1}\right) C_{N_{1}} \cdots \cdots C_{N_{n}}\left\|\varphi_{1}\right\| \cdots \cdots\left\|\varphi_{n}\right\| .
\end{aligned}
$$

We use a formula of Dudley Johnson [2, p. 282];

$$
\begin{aligned}
& \sum_{i_{n=1}}^{n} \sum_{i_{n-1}=1}^{n-1} \cdots \cdots \sum_{i_{1}=1}^{1} \tau_{n}\left(i_{1}, \cdots \cdots, i_{n}\right) ! \cdots \cdots \tau_{1}\left(i_{1}, \cdots \cdots, i_{n}\right) ! \\
& \quad=1.3 \cdots \cdots(2 n-1) \leq 2^{n} \cdot n !
\end{aligned}
$$

where $\tau_{k}\left(i_{1}, \cdots \cdots, i_{n}\right)$ is the number of $i_{1}, \cdots \cdots, i_{n}$ which are equal to $k$.

$$
\begin{aligned}
& \left\|D \varphi_{n} \cdots \cdots D \varphi_{1}\right\| \\
& \quad \leq \sum_{n=0}^{\infty} \cdots \cdots \sum_{N_{1}=0}^{\infty}\left\|Q\left(N_{n}\right) \varphi_{n} \cdots \cdots Q\left(N_{1}\right) \varphi_{1}\right\| \\
& \quad \leq \sum_{n=0}^{\infty} \cdots \cdots \sum_{N_{1}=0}^{\infty} M\left(M+N_{1}\right) \cdots\left(M+N_{1}+\cdots+N_{n-1}\right) C_{N_{1}} \cdots C_{N_{n}}\left\|\varphi_{1}\right\| \cdots\left\|\varphi_{n}\right\| \\
& \quad \leq(M+1)^{n}\left\|\varphi_{1}\right\| \cdots \cdots\left\|\varphi_{n}\right\| \sum_{N_{n}=0}^{\infty} \cdots \cdots \sum_{N_{1}=0}^{\infty}\left(\sum_{i_{n}=1}^{n} N_{i_{n}}+1\right)\left(\sum_{i_{n-1}=1}^{n-1} N_{i_{n-1}}+1\right) \cdots \cdots \\
& \quad \ldots \cdots\left(\sum_{i_{1}=1}^{1} N_{i_{1}}+1\right) C_{N_{1}} \cdots \cdots C_{N_{n}} \\
& =(M+1)^{n}\left\|\varphi_{1}\right\| \cdots \cdots\left\|\varphi_{n}\right\| \sum_{N_{n}=0}^{\infty} \cdots \cdots \sum_{N_{1}=0}^{\infty} \sum_{i_{n}=0}^{n} \cdots \cdots \sum_{i_{0}=0}^{1} N_{i_{n}} \cdots \cdots N_{i_{1}} C_{N_{1}} \cdots \cdots C_{N_{n}}
\end{aligned}
$$

(where $N_{0} \equiv 1$ ) 


$$
\begin{aligned}
& =(M+1)^{n}\left\|\varphi_{1}\right\| \cdots \cdots \cdot \varphi_{n} \| \sum_{i_{n}=0}^{n} \cdots \cdots \sum_{i_{1}=0}^{1}\left(\sum_{N_{n}=0}^{\infty} N_{n}^{\tau_{n}\left(i_{1}, \cdots, i_{n}\right)} C_{N_{n}}\right) \cdots \cdots \\
& \cdots \cdots\left(\sum_{N_{1}=0}^{\infty} N_{1}^{\tau_{1}\left(i_{1}, \ldots, i_{n}\right)} C_{N_{1}}\right) \\
& \leq(M+1)^{n}\left\|\varphi_{1}\right\| \cdots \cdots\left\|\varphi_{n}\right\| \sum_{i_{n}=0}^{n} \cdots \cdots \sum_{i_{1}=0}^{1} \tau_{n}\left(i_{1} \cdots \cdots i_{n}\right) ! L^{\tau_{n}\left(i_{1}, \ldots, i_{n}\right)} \\
& \cdots \cdots \tau_{1}\left(i_{1}, \cdots \cdots, i_{n}\right) ! L^{\tau_{1}\left(i_{1}, \ldots, i_{n}\right)} \tau_{0}\left(i_{1}, \cdots \cdots, i_{n}\right) ! L^{\tau_{0}\left(i_{1}, \cdots, i_{n}\right)} \\
& \leq(M+1)^{n}\left\|\varphi_{1}\right\| \cdots \cdots\left\|\varphi_{n}\right\| L^{n} \sum_{i_{n}=0}^{n} \cdots \cdots \sum_{i_{0}=0}^{0} \tau_{n}\left(i_{0}, \cdots \cdots, i_{n}\right) ! \\
& \cdots \cdots \tau_{1}\left(i_{0}, \cdots \cdots, i_{n}\right) ! \tau_{0}\left(i_{0}, \cdots \cdots, i_{n}\right) !
\end{aligned}
$$

(by appyling (2.12) here)

$$
\leq(M+1)^{n} L^{n} \cdot 2^{n+1} \cdot(n+1) !\left\|\varphi_{1}\right\| \cdots \cdots\left\|\varphi_{n}\right\| .
$$

Changing the order of indices, we have

$$
\left\|D \varphi_{1} \cdots \cdots D \varphi_{n}\right\| \leq 2(n+1) !(2(M+1) L)^{n}\left\|\varphi_{1}\right\| \cdots \cdots\left\|\varphi_{n}\right\| .
$$

Thus, (2.10) is obtained, noting that

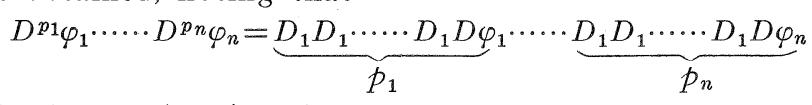

(2.11) is obtained from (2.10) and the following:

$$
\begin{aligned}
& \left\|e^{t_{1} D} \varphi_{1} \cdots \cdots e^{t_{n} D} \varphi_{n}\right\| \leq \sum_{p_{1}=0}^{\infty} \cdots \cdots \sum_{p_{n}=0}^{\infty} \frac{t_{1}^{p_{1} \cdots \cdots t_{n} p_{n}}}{p ! \cdots \cdots p_{n} !}\left\|D^{p_{1}} \varphi_{1} \cdots \cdots D^{p_{n}} \varphi_{n}\right\|
\end{aligned}
$$

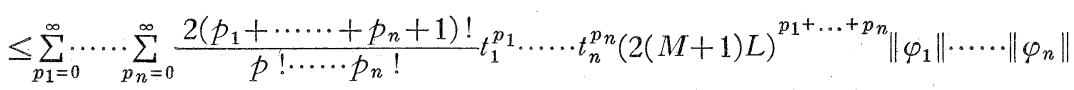

$$
\begin{aligned}
& =2 \sum_{N=0}^{\infty}(N+1)(2(M+1) L)^{N}\left(t_{1}+\cdots \cdots+t_{n}\right)^{N}\|\varphi\| \cdots \cdots \cdot\left\|\varphi_{n}\right\| \\
& =2\left(1-2(M+1) L\left(t_{1}+\cdots \cdots+t_{n}\right)\right)^{-2}\left\|\varphi_{1}\right\| \cdots \cdots\left\|\varphi_{n}\right\|, \\
& \text { if } t_{1}+\cdots \cdots+t_{n}<(2(M+1) L)^{-1} \text {. }
\end{aligned}
$$

\section{$\S 3$. The motion of one particle}

We assume, for simplicity, that $\{1,2, \cdots \cdots\}$ is contained in $I$.

LEMma 3. For a probability measure $f$ on $(S, \boldsymbol{B}(S))$ and a function $\varphi \in F_{0}$ of one variable $x \equiv x_{1}$ where $1 \in I_{0}$,

$$
\begin{aligned}
& \int_{\{1\}^{c}} f^{\infty} D \varphi=A^{f} \varphi(x) \\
& \int f^{\infty} D \varphi=\int_{S} f(d x) A^{f} \varphi(x) .
\end{aligned}
$$

For $t<1 / 4 L$, there are probability measures $P^{f}(t, x, \cdot)$ and $f_{t}(\cdot)$ on $(S, \boldsymbol{B}(S))$ such that

$$
\begin{aligned}
& \int_{\{1\}} f^{\infty} e^{t D} \varphi=\int_{S} P^{f}(t, x, d y) \varphi(y), \\
& \int f^{\infty} e^{t D} \varphi=\int_{S} f_{t}(d x) \varphi(x) .
\end{aligned}
$$

In general, for subsets $J=\{1,2, \cdots \cdots, k\}, J_{1}=\{1,2, \cdots \cdots, h\}$ of $\left.I_{0} *\right)$ with $h \leq k$ and

*) Note that $I_{0}$ can be chosen arbitrarily on which the definitions of $D$ and $e^{t D}$ depend. 
$t<1 / 2 L(k+1)$, there are probability measures $P_{J}^{f}\left(t_{1}, x_{1}, \cdots \cdots, x_{h}, \cdot\right)$ and $f_{t, J}(\cdot)$ on $\left(S^{J}, \boldsymbol{B}\left(S^{J}\right)\right)$ such that

$$
\begin{aligned}
& \int_{J_{1}^{c}} f^{\infty} e^{t D} \varphi=\int_{S J} P_{J}^{f}\left(t, x_{1}, \cdots \cdots, x_{h}, d x_{1}, \cdots \cdots, d x_{k}\right) \varphi\left(x_{1}, \cdots \cdots, x_{k}\right) \\
& \int f^{\infty} e^{t D} \varphi=\int_{S J} f_{t}, J\left(d x_{1}, \cdots \cdots, d x_{k}\right) \varphi\left(x_{1}, \cdots \cdots, x_{k}\right)
\end{aligned}
$$

for any function $\varphi$ in $F_{0}$ with $I(\varphi)=J . \quad P_{J}^{f}\left(t, x_{1}, \cdots \cdots, x_{h}, E\right)$ is measurable in $\left(x_{1}\right.$, $\left.\cdots \cdots, x_{h}\right)$ for any $E \in \boldsymbol{B}\left(S^{J}\right)$, and

$$
f_{t, J}(E)=\int_{S^{J_{1}}} f\left(d x_{1}\right) \cdots \cdots f\left(d x_{h}\right) P_{J}^{f}\left(t, x_{1}, \cdots \cdots, x_{h}, E\right) .
$$

Proposition 1.*) Let $m$ and $q(x, \cdot)$ be signed measures on measurable spaces $(R, \boldsymbol{B}(R))$ and $(S, \boldsymbol{B}(S))$ with finite total variations $\|m\|$ and $\|q\|(x)$ respectively, and let $q(x, E)$ be a $\boldsymbol{B}(R)$. measurable function on $R$ for each fixed $E \in \boldsymbol{B}(S)$. If $\int_{R}|m|(d x)\|q\|(x)<\infty$, then, the set function $\int m(d x) q(x, E), \quad E \in \boldsymbol{B}(S), \quad$ is a signed measure on $(S, \boldsymbol{B}(S))$ with total variation at most $\int|m|(d x)\|q\|(x)$.

Proof of Proposition. In order that $\int m(d x) q(x, E)$ is a measure, it is sufficient to prove

$$
\int m(d x) \int q(x, d y)\left(\sum_{n} \varphi_{n}(y)\right)=\sum_{n} \int m(d x) \int q(x, d y) \varphi_{n}(y)
$$

if $\sum\left|\varphi_{n}(x)\right| \leq k<\infty$ and $\varphi_{n}$ are measurable. But, by bounded convergence theorem the left side is equal to

$$
\int m(d x)\left(\sum_{n} \int q(x, d y) \varphi_{n}(y)\right)
$$

Since $\sum_{h=1}^{n} \int q(x, d y) \varphi_{h}(y)$ is convergent for each $x$ and bounded by $k\|q\|$, we can apply the bounded convergence theorem again, and have the equality. Total variation of this measure is

$$
\begin{aligned}
\int m(d x) q(x, & \left.E^{+}\right)-\int m(d x) q\left(x, E^{-}\right) \\
& =\int m(d x)\left(q\left(x, E^{+}\right)-q\left(x, E^{-}\right)\right) \leq \int|m|(d x)\left|q\left(x, E_{x}^{+}\right)-q\left(x, E_{x}^{-}\right)\right| \\
& =\int|m|(d x)\|q\|(x),
\end{aligned}
$$

where $E^{+}$and $E^{-}$, and $E_{x}^{+}$and $E_{x}^{-}$are the positive set and the negative set of $\int m(x, d y) q(x, \cdot)$ and of $q(x, \cdot)$, respectively.

Proof of Lemma 3. Since $\|D \varphi\| \leq \sum_{N=0}^{\infty}\|Q(N) \varphi\|<\infty$ for $\varphi \in F_{0}$,

$$
\int_{\{1\}}^{c} f^{\infty} D \varphi=\int_{\{1\}} f^{\infty} \sum_{N=0}^{\infty} Q(N) \varphi=\sum_{N=0}^{\infty} \int_{\{1\}} f^{\infty} Q(N) \varphi
$$

*) Note that this is also true for complex valued measures with finite total variation. 
For $\varphi$ with indices in $J$,

$$
\begin{array}{r}
=\sum_{N=0}^{\infty} \int_{i=2}^{N+1} \prod_{i=2} f\left(d x_{i}\right) A_{N} \varphi\left(x_{2}, \cdots \cdots, x_{N+1} \mid x_{1}\right) \\
=A^{f} \varphi\left(x_{1}\right) \\
\int f^{\infty} D \varphi_{1}=\int_{S} f(d x) \int_{\{1\}}^{c} f^{\infty} D \varphi=\int_{S} f(d x) A^{f} \varphi(x) .
\end{array}
$$

$$
Q(N) \varphi=\sum_{i \in I(\varphi)} A_{N}^{(i)} \varphi=\sum_{i=1}^{k} \int_{S} A_{N}\left(x_{i_{1}}, \cdots \cdots, x_{i_{N}} \mid x_{i} ; d y\right) \varphi\left(x_{1}, \cdots, \stackrel{i}{i}, \ldots \ldots, x_{k}\right)
$$

by definition. Hence, $Q(N) \varphi$ is clearly an integral of $\varphi$ by a signed measure on $\left(S^{J}\right.$, $\left.\boldsymbol{B}\left(S^{J}\right)\right)$. Similarly, with Proposition in mind, we know that $Q\left(N_{n}\right) \cdots \cdots Q\left(N_{1}\right) \varphi$ is an integral of $\varphi$ by a signed measure on $\left(S^{J}, \boldsymbol{B}\left(S^{J}\right)\right)$ with total variation at most $\left(N_{n-1}+\cdots \cdots+N_{1}+k\right) \cdots \cdots\left(N_{1}+k\right) k C_{N_{n}} \cdots \cdots C_{N_{1}}$ indexed by $\left(N_{n}+\cdots \cdots+N_{1}+k\right)$ variables. And hence,

$$
\int_{J_{1}^{c}} f^{\infty} Q\left(N_{n}\right) \cdots \cdots Q\left(N_{1}\right) \varphi
$$

is also given by a signed measure on $\left(S^{J}, \boldsymbol{B}\left(S^{J}\right)\right)$ with the total variation bounded by $\left(N_{n-1}+\cdots \cdots+N_{1}+k\right) \cdots \cdots\left(N_{1}+k\right) k C_{N_{n}} \cdots \cdots C_{N_{1}}$. Since

$$
\sum_{n=0}^{\infty} \frac{t^{n}}{n !} \sum_{N_{n}=0}^{\infty} \cdots \cdots \sum_{N_{1}=0}^{\infty}\left(N_{n-1}+\cdots \cdots+N_{1}+k\right) \cdots \cdots\left(N_{1}+k\right) k C_{N_{n}} \cdots \cdots C_{N_{1}}
$$

is convergent for $t<1 / 2 L(k+1)$ as we saw in the proof of Lemma 2, the following is also given by a signed measure on $\left(S^{J}, \boldsymbol{B}\left(S^{J}\right)\right)$ indexed by $x_{1}, \cdots \cdots, x_{h}$ which we denote by $P_{J}^{f}\left(t, x_{1}, \cdots \cdots, x_{h}, \cdot\right)$.

$$
\sum_{n=0}^{\infty} \frac{t^{n}}{n !} \sum_{N_{n}=0}^{\infty} \cdots \cdots \sum_{N_{1}=0}^{\infty} \int_{J_{1}^{c}} f^{\infty} Q\left(N_{n}\right) \cdots \cdots Q\left(N_{1}\right) \varphi .
$$

But, this is clearly equal to

$$
\int_{J_{1}^{c}} f^{\infty} \sum_{n=0}^{\infty} \frac{t^{n}}{n !} \sum_{N_{1}=0}^{\infty} \cdots \cdots \sum_{N_{n}=0}^{\infty} Q\left(N_{n}\right) \cdots \cdots Q\left(N_{1}\right) \varphi=\int_{J_{1}^{c}} f^{\infty} e^{t D} \varphi .
$$

$\left(3.3^{\prime}\right)$ is obtained by replacing $\int_{J_{1}^{c}}$ by $\int$, and denoting the correponding measure by $f_{t, J},(3.4)$ is clear, since

$$
\int f^{\infty} \psi=\int_{S^{J_{1}}} f\left(d x_{1}\right) \cdots \cdot f\left(d x_{h}\right) \int_{J_{1}^{c}} f^{\infty} \psi
$$

That the measures $P_{J}^{f}\left(t, x, \cdots \cdots, x_{h}, \cdot\right)$ and $f_{t, J}(\cdot)$ are non-negative with total mass 1 , will be proved in the Corollary to Theorem 4.

Lemma 4. Assume that $e^{t D} \varphi$ can be defined for $\varphi \in F$ and $x_{1}$ is a variable of $\varphi$. Then,

$$
\int_{[1\}}^{c} f^{\infty} e^{t D} \varphi=\int_{\{1\}}^{c} f^{\infty} e^{t D}\left(\int_{\{1\}}^{c} f_{t}^{\infty} \varphi\right)
$$

REMARK. We can replace $\{1\}^{c}$ by $J^{c}$ in Lemma 4 , where $J$ is a finite set contained in $I(\varphi)$. Proof is similar, replacing $\{1\}$ by $J$.

Proof. First, we consider the following case. 
(3.6)

$$
\varphi=\varphi\left(x_{1}, \cdots \cdots, x_{k}\right)=\varphi_{1}\left(x_{1}\right) \cdots \cdots \varphi_{k}\left(x_{k}\right) .
$$

Then, (3.5) is true by (2.6) of Lemma and (3.2') of Lemma 3 :

$$
\begin{aligned}
\int_{\{1\}^{c}} f^{\infty} e^{t D} \varphi & =\int_{\{1\}} c f^{\infty} e^{t D}\left(\varphi_{1} \otimes \cdots \cdots \otimes \varphi_{k}\right) \\
& =\int_{\{1\}} f^{\infty}\left(e^{t D} \varphi_{1}\right) \otimes \cdots \cdots \otimes\left(e^{t D} \varphi_{k}\right)=\int_{I\left(e^{\left.t D \varphi_{1}\right)-\{1\}}\right.} f^{\infty} e^{t D} \varphi_{1} \cdot \prod_{i=2}^{k} \int_{I\left(e^{t} t \varphi_{i}\right)} f^{\infty} e^{t D} \varphi_{i} \\
& =\int_{\{1\}} c f^{\infty} e^{t D} \varphi_{1} \cdot \prod_{i=2}^{k} \int f^{\infty} e^{t D} \varphi_{i}=\int_{\{1\}} f^{\infty} e^{t D} \varphi_{1} \cdot \prod_{i=2}^{k} \int_{S} f_{t}\left(d x_{i}\right) \varphi_{i}\left(x_{i}\right) \\
& =\int_{\{1\}}^{c} f^{\infty} e^{t D} \varphi_{1} \cdot \int f_{t}^{\infty} \varphi_{2} \cdots \cdots \varphi_{k}=\int_{\{1\}^{c}} f^{\infty} e^{t D}\left(\int_{\{1\}}^{c} f_{t}^{\infty} \varphi_{1} \cdots \cdots \varphi_{k}\right) \\
& =\int_{\{1\}}^{c} f^{\infty} e^{t D} \int_{\{1\}}^{c} f_{t}^{\infty} \varphi .
\end{aligned}
$$

Hence, (3.5) also holds for linear combinations of functions of type (3.6). On the other hand, for $\varphi$ in $F_{0},(3.5)$ is equivalent to

$$
\begin{aligned}
& \int_{S^{J}} P_{J}^{f}\left(t, x_{1} ; d y_{1}, \cdots \cdots, d y_{k}\right) \varphi\left(y_{1}, \cdots \cdots, y_{k}\right) \\
& \quad=\int_{S} P^{f}\left(t, x_{1}, d y_{1}\right) \int_{S^{J}-\{1\}} f_{t}\left(d y_{2}\right) \cdots \cdots f_{t}\left(d y_{k}\right) \varphi\left(y_{1}, \cdots \cdots, y_{k}\right),
\end{aligned}
$$

by (3.2) and of Lemma 3 taking $\{1\}$ as $J_{1}$ and $\{1,2, \cdots \cdots, k\}$ as $J$. Thus, by a standard technique of measure theory, (3.5) is true for all $\varphi \in F_{0}{ }^{*}$.

For $\varphi$ in $F$ for which $e^{t D} \varphi$ exists, there is a sequence $\left\{\varphi_{n}\right\} \subset F_{0}$, such that $\sum_{1}^{n} \varphi_{j}$ and $e^{t D}\left(\sum_{1}^{n} \varphi_{j}\right)$ converge to $\varphi$ and $e^{t D} \varphi$ in norm for any fixed choice of variables, respectively. Then, the bounded convergence theorem applies to both sides of (3.5), completing the proof.

REMARK. $P_{J}^{f}\left(t, x_{1}, \cdots \cdots, x_{h}, d y_{1}, \cdots \cdots, d y_{k}\right)$ in Lemma 3 is the product measure

$$
\prod_{i \in J_{1}} P^{f}\left(t, x_{i}, d y_{i}\right) \times \prod_{j \in J-J_{1}} f_{t}\left(d y_{j}\right)
$$

$\left(3.5^{\prime}\right)$ is a special case when $J_{1}=\{1\}$. This comes from the property (2.6) of exponential $e^{t D}$.

LEMMA 5. For functions $\varphi_{1}, \varphi_{2}, \cdots \cdots, \varphi_{n} \in F_{0}$ of one common variable $x_{1} \equiv x$, and for $t_{1}+\cdots \cdots+t_{n}<1 / 4 L$,

$$
\begin{aligned}
& \int_{\{1\}}^{c} f^{\infty} e^{t_{1} D} \varphi_{1} e^{t_{2} D} \varphi_{2} \cdots \cdots e^{t_{n} D} \varphi_{n} \\
& \quad=\int_{S} P^{f}\left(t_{1}, x, d x_{1}\right) \varphi_{1}\left(x_{1}\right) \int_{S} P^{f t_{1}}\left(t_{2}, x_{1}, d x\right) \varphi_{2}\left(x_{2}\right) \cdots \cdots \\
& \quad \cdots \cdots \int_{S} P^{f t_{1}+\cdots+t_{n-1}}\left(t_{n}, x_{n-1}, d x_{n}\right) \varphi_{n}\left(x_{n}\right), \\
& \int f^{\infty} e^{t_{1} D} \varphi_{1} \cdots \cdots e^{t_{n} D_{n}} \varphi_{n} \\
& \quad=\int_{S} f(d x) P^{f}\left(t_{1}, x, d x_{1}\right) \varphi_{1}\left(x_{1}\right) \int_{S} \cdots \cdots \int_{S} P^{f t_{1}+\cdots+t n}\left(t_{n}, x_{n-1}, d x_{n}\right) \varphi_{n}\left(x_{n}\right) . \\
& P^{f}(s+t, x, \cdot)=\int_{S} P^{f}(s, x, d y) P^{f s}(t, y, \cdot),
\end{aligned}
$$

*) The reader can apply, for instance, I. T 20 of Meyer : Probability and Potentials. 


$$
f_{s+t}=(f s)_{t}, \text { for } s+t<1 / 4 L \text {. }
$$

Proof. Applying (3.5) of Lemma 4 to $\varphi_{i} e^{t_{i+1} D} \varphi_{i+1} \cdots \cdots e^{t_{n} D} \varphi_{n}$ $(1 \leq i<n)$ repeatedly, we have

$$
\begin{aligned}
& \int_{\{1\}}^{c} f^{\infty} e^{t_{1} D} \varphi_{1} e^{t_{2} D} \varphi_{2} \cdots \cdots e^{t_{n} D} \varphi_{n}=\int_{\{1\}} f^{\infty} e^{t_{1} D} \int_{\{1\}} f^{c} f_{t_{1}}^{\infty} \varphi_{1} e^{t_{2} D} \varphi_{2} \cdots \cdots e^{t_{n} D} \varphi_{n} \\
& =\int_{\{1\}}^{c} f^{\infty} e^{t_{1} D} \varphi_{1} \int_{\{1\}} f_{t_{1}}^{\infty} e^{t_{2} D} \varphi_{2} \cdots \cdots e^{t_{n} D} \varphi_{n} \\
& =\int_{\{1\}}^{c} f^{\infty} e^{t_{1} D} \varphi_{1} \int_{\{1\}} c f_{t_{1}}^{\infty} e^{t_{2} D} \varphi_{2} \int_{\{1\}} c\left(f_{t_{1}}\right)_{t_{2}}^{\infty} e^{t_{3} D} \varphi_{3} \cdots \cdots e^{t_{n} D} \varphi_{n} \\
& =\int_{\{1\}}^{c} f^{\infty} e^{t_{1} D} \varphi_{1} \int_{\{1\}} c^{c} \cdots \cdot \int_{\{1\}}^{c}\left(\left(f_{t_{1}}\right) t_{2} \cdots \cdots\right)_{t_{n-1}}^{\infty} e^{t_{n} D} \varphi_{n} \\
& =\int_{S} P^{f}\left(t_{1}, x, d x\right) \varphi_{1}\left(x_{1}\right) \int_{S} P^{f t_{1}}\left(t_{2}, x_{1}, d x_{2}\right) \varphi_{2}\left(x_{2}\right) \int_{S} \cdots \cdots \\
& \cdots \cdots \int_{S} P^{\left(\cdots\left(f_{t_{1}}\right) t_{2} \cdots\right)_{t_{n-1}}}\left(t_{n}, x_{n-1}, d x_{n}\right) \varphi_{n}\left(x_{n}\right),
\end{aligned}
$$

where the last equality sign owes to Lemma 3. The proof of (3.7) is complete, if $\left(3.8^{\prime}\right)$ is proved. $\left(3.7^{\prime}\right)$ is obtained by integrating the both sides of (3.7) by $f$.

(3.8) is proved by (2.7) of Lemma 1 and Lemma 4 .

$$
\begin{aligned}
\int_{S} P^{f}(s+t & , x, d y) \varphi(y)=\int_{\{1\}} f^{\infty} e^{(s+t) D} \varphi \\
& =\int_{\{1\}}{ }^{c} f^{\infty} e^{s D} e^{t D} \varphi=\int_{\{1\}}^{c} f^{\infty} e^{s D} \int_{\{1\}^{c}} f_{s}^{\infty} e^{t D} \varphi \\
& =\int_{\{1\}}^{c} f^{\infty} e^{s D} \int_{S} P^{f s}\left(t, x_{1}, d z\right) \varphi(z) \\
& =\int_{S} P^{f}(s, x, d x) \int_{S} P^{f s}\left(t, x_{1}, d z\right) \varphi(z) .
\end{aligned}
$$

$\left(3.8^{\prime}\right)$ is clear by integrating (3.8) by $f$.

Now, we are in the position to define $P^{f}(t, x, \cdot)$ and $f_{t}(\cdot)$ for an arbitrary $t>0$. In fact, for any $t>0$, take a sequence $t_{1}, \cdots \cdots, t_{n}>0$ such that

and define

$$
t=t_{1}+\cdots \cdots+t_{n} \text {, and } t_{k}<1 / 4 L, k=1, \cdots \cdots, n,
$$

$$
\begin{aligned}
P^{f}(t, x, \cdot) & \left.=\int_{S} P^{f}\left(t_{1}, x, d x_{1}\right) \int_{S} P^{f_{t_{1}}\left(t_{2}, x_{1}\right.}, d x_{2}\right) \int_{S} \cdots \cdots \\
& \cdots \cdots \int_{S} P^{\left(\cdots\left(f_{t_{1}}\right)_{t_{2}} \cdots\right) t_{n-2},}\left(t_{n-1}, x_{n-2}, d x_{n-1}\right) P^{\left(\cdots\left(f_{t_{1}}\right)_{t_{2}} \cdots\right)_{t_{n-1}}\left(t_{n}, x_{n-1}, \cdot\right)} \\
f_{t}(\cdot)= & \int_{S} f(d x) P^{f}(t, x, \cdot)
\end{aligned}
$$

By virtue of Chapman-Kolmogorov equation (3.8) for small $t$, it is easy to prove that this is independent of the choice of $t_{1}, \cdots \cdots, t_{n}$. And it is clear that (3.8) and $\left(3.8^{\prime}\right)$ hold true for arbitrary $s$ and $t$.

Proof of Theorem 1. Chapman-Kolmogorov equation has been discussed above. For $t<\frac{1}{4 L}$, we have

$$
\begin{gathered}
\frac{1}{t}\left(\int_{S} P^{f}(t, x, d y) \varphi(y)-\varphi(x)\right)=\frac{1}{t}\left(\int_{\{1\}} f^{\infty} e^{t D} \varphi(x)-\varphi(x)\right) \\
=\frac{1}{t}\left(\sum_{n=0}^{\infty} \frac{t^{n}}{n !} \int_{\{1\}} f^{\infty}\left(D^{n} \varphi(x)-\varphi(x)\right)\right.
\end{gathered}
$$




$$
=\int_{\{1\}^{c}} f^{\infty} D \varphi(x)+\frac{1}{t} \sum_{n=2}^{\infty} \frac{t^{n}}{n !} \int_{\{1\}} f^{\infty} D^{n} \varphi(x),
$$

by Lemma 3. But, the second term is bounded by

$$
\begin{aligned}
\frac{1}{t} \sum_{n=2}^{\infty} \frac{t^{n}}{n !} \int_{\{1\}^{c}} f^{\infty}\left\|D^{n} \varphi\right\| \leq \frac{2}{t} \sum_{n=2}^{\infty} \frac{t^{n}}{n !} \cdot(n+1) !(2(M+1) L)^{n}\|\varphi\| \\
=\frac{2}{t} \sum_{n=2}^{\infty} t^{n}(n+1)(4 L)^{n}\|\varphi\|=\frac{2}{t}\left(\frac{1}{(1-4 t L)^{2}}-1-8 t L\right)\|\varphi\|
\end{aligned}
$$

and hence converges to 0 as $t \rightarrow 0$, where the estimate of $\left\|D^{n} \varphi\right\|$ is given in Lemma 2. Thus, the left-hand side of (1.5) converges to $\int_{\{1\}} f^{\infty} D \varphi(x)$ uniformly in $x$ which is equal to $A^{f} \varphi(x)$ by (3.1) of Lemma 3 .

Since the convergence of (1.5) is uniform in $x$, the right derivative (in $t$ ) of $\int_{S} P^{f}(t, x, d y) \varphi(y)$ exists and is equal to $\int_{S} P^{f}(t, x, d y) A^{f t} \varphi(y)$, by

$$
\begin{aligned}
\frac{1}{\Delta}\left(\int_{S} P^{f}(t+\Delta, x, d y) \varphi(y)-\int_{S} P^{f}(t, x, d y) \varphi(y)\right. \\
\quad=\int_{S} P^{f}(t, x, d y) \frac{1}{\Delta}\left(\int_{S} P^{f t}(\Delta, y, d z) \varphi(z)-\varphi(y)\right) .
\end{aligned}
$$

In order that it is also the derivative from the left (in case $t>0$ ), it is sufficient to prove (1.7). But, this follows from

$$
\begin{aligned}
& \int_{S} P^{f}(s+t, x, d y) \varphi(y)=\int_{S} P^{f}(s, x, d y) \int_{S} P^{f} s(t, y, d z) \varphi(z) \\
& =\sum_{n=0}^{\infty} \frac{t^{n}}{n !} \int_{S} P^{f}(s, x, d y) \int_{\{1\}^{c}} f_{\delta}^{\infty} D^{n} \varphi(y) \text {, for } t<1 / 4 L,
\end{aligned}
$$

where the coefficient of $t^{n}$ is bounded by $(n+1)(4 L)^{n}\|\varphi\|$ for any $s>0$, completing the proof.

Let $W$ be the set of all $S$-valued functions defined on $[0, \infty)$, that is, $S^{[0, \infty)}$. Let $\boldsymbol{B}=\boldsymbol{B}(W)$ be the $\sigma$-algebra of subsets of $W$ generated by all cylinder sets. $P_{x}^{f}$ is the probability measure on $(W, \boldsymbol{B})$ given as the unique extensions of a set function for cylinder sets:

$$
\begin{aligned}
& P_{x}^{f}\left(w t_{1} \in A_{1}, \cdots \cdots, w t_{n} \in A_{n}\right) \\
& =\int_{A_{1}} P^{f}\left(t, x, d x_{1}\right) \int_{A_{2}} P^{f t_{1}}\left(t_{2}-t_{1}, x_{1}, d x_{2}\right) \int_{A_{3}} \cdots \cdots \int_{A_{n}} P^{f t_{n-2}\left(t_{n-1}-t_{n-2}, x_{n-2}, d x_{n-1}\right)}
\end{aligned}
$$

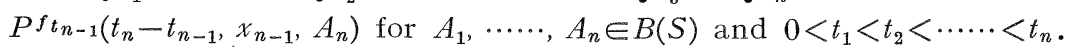

$$
\begin{aligned}
& P^{f}(\Lambda)=\int_{S} f(d x) P_{x}^{f}(\Lambda) .
\end{aligned}
$$

$E_{x}^{f}$ and $E^{f}$ are expectations by $P_{x}^{f}$ and $P^{f}$, respectively. We write the value of $w$ at time $t$ as $w_{t}$ or $x_{t}(w)$. Then, $\left(W, \boldsymbol{B}, P^{f}, x_{t_{n-1}}\right)$ is a temporally inhomogeneous Markov process with initial distribution $f$ and transition probability

since

$$
P(s, x, t, E)=P^{f} s(t-s, x, E), s<t,
$$

$$
\begin{aligned}
P^{f}\left(x_{t_{1}} \in\right. & \left.A_{1}, \cdots \cdots, x_{t_{n}} \in A_{n} / \boldsymbol{B}_{s}\right) \\
& =P_{x_{s}}^{f_{s}}\left(x_{t_{1-s}} \in A_{1}, \cdots \cdots, x_{t_{n-s}} \in A_{n}\right) \\
& =P^{f}\left(x_{t_{1}} \in A_{1}, \cdots \cdots, x_{t_{n}} \in A_{n} / \boldsymbol{B}\left(x_{s}\right)\right), P^{f}-a . e . .
\end{aligned}
$$


Here, $\boldsymbol{B}_{\boldsymbol{s}}$ and $\boldsymbol{B}\left(x_{\boldsymbol{s}}\right)$ are the $\sigma$-subalgebra of $\boldsymbol{B}$ generated by the cylinder sets of type $\left\{x_{r_{1}} \in A_{1}, \cdots \cdots, x_{r_{n}} \in A_{n}\right\}, r_{1}, \cdots \cdots, r_{n} \leq s$, and $\left\{\chi_{s} \in A\right\}$, respectively.

By these notations, a part of Lemma 5 is restated in the following way.

THEOREM 3. For functions $\varphi_{1}, \cdots \cdots, \varphi_{n}$ in $F_{0}$ of one variable and for $t_{1}, \cdots \cdots, t_{n}$ satisfying $0<t_{1}<\cdots \cdots<t_{n}<1 / 4 L$,

$$
\begin{aligned}
& E^{f}\left(\varphi_{1}\left(x_{t_{1}}\right) \cdots \cdots \varphi_{n}\left(x_{t_{n}}\right)\right)=\int f^{\infty} e^{t_{1} D} \varphi_{1}, \cdots \cdots, e^{\left(t_{n}-t_{n-1}\right) D} \varphi_{n}, \\
& E_{x}^{f}\left(\varphi_{1}\left(x_{1}\right) \cdots \cdots \varphi_{n}\left(x_{t_{n}}\right)\right)=\int_{\{1\}} f^{\infty} e^{t_{1} D} \varphi_{1}, \cdots \cdots, e^{\left(t_{n}-t_{n-1}\right) D} \varphi_{n} .
\end{aligned}
$$

\section{$\S 4$. Propagation of chaos}

First, we note that $G_{n}$ is bounded, that is,

$$
\begin{aligned}
\left\|G_{n}\right\| & =\sup _{\left(x_{1}, \ldots, x_{n}\right)} \text { total variation of } G_{n}\left(x_{1}, \cdots \cdots, x_{n} ; \cdot\right) \\
& \leq \sum_{N=0}^{n-1} n^{-N} \sum_{\left(j_{1}, \ldots, j_{N}, i\right)}^{(n)}\left\|A_{N}^{(i)}\left(x_{j_{1}}, \cdots \cdots, x_{j_{N}}\right)\right\|=\sum_{N=0}^{n-1} n^{-N} \sum_{\left(j_{1}, \ldots, j_{N}, i\right)}^{(n)} C_{N} \\
& =\sum_{N=0}^{n-1} n^{-N}\left(\begin{array}{c}
n \\
N+1
\end{array}\right) C_{N}=\sum_{N=0}^{n-1} n^{-N} \frac{n !}{(n-N-1) !(N+1) !} C_{N} \leq n \sum_{N=0}^{n-1} \frac{C_{N}}{(N+1) !} \leq n \sum_{N=0}^{\infty} C_{N} \leq n L
\end{aligned}
$$

Moreover, by looking at the definition (1.9) carefully, we know that $G_{n}$ is a stochastic generator kernel, that is,

$$
\begin{gathered}
G_{n} \varphi\left(x_{1}, \cdots, x_{n}\right)=q_{n}\left(x_{1}, \cdots \cdots, x_{n}\right) \int_{S_{n}} \pi_{n}\left(x_{1}, \cdots \cdots, x_{n} ; d y_{1}, \cdots, d y_{n}\right) \\
\left(\varphi\left(y_{1}, \cdots \cdots, y_{n}\right)-\varphi\left(x_{1}, \cdots \cdots, x_{n}\right)\right),
\end{gathered}
$$

where $\pi_{n}$ is a probability measure on $\left(S^{n}, \boldsymbol{B}\left(S^{n}\right)\right)$ and $q_{n}$ is non-negative. Hence, $e^{t G_{n}}$ defines a non-negative, strongly continuous semi-group on $B\left(S^{n}\right)$ and $e^{t G_{n}} 1=1$. Then, there is a probability measure on $P_{n}\left(t, x_{1}, \cdots \cdots, x_{n} ; \cdot\right)$ on $\left(S^{n}, \boldsymbol{B}\left(S^{n}\right)\right)$ such that

$$
e^{t G n} \varphi\left(x_{1}, \cdots \cdots, x_{n}\right)=\int_{S^{n}} P_{n}\left(t, x_{1}, \cdots \cdots, x_{n} ; d y_{1}, \cdots \cdots, d y_{n}\right) \varphi\left(y_{1}, \cdots \cdots, y_{n}\right) \in B\left(S^{n}\right)
$$

Thus, the construction of $M_{n}$ in $\S 1$ is clearly possible. We introduce $Q_{n}(N)$ on $B\left(S^{n}\right)$ by

Then,

$$
\begin{gathered}
Q_{n}(N) \varphi\left(x_{1}, \cdots \cdots, x_{n}\right)=n^{-N} \sum_{\left(j_{1}, \ldots, j_{N}, i\right)}^{(n)} \int_{S} A_{N}\left(x_{j_{1}}, \cdots \cdots, x_{j_{N}} \mid x_{i}, d y\right) \\
\varphi(x_{1}, \cdots \cdots, \overbrace{y}^{i}, \cdots \cdots, x_{n}) .
\end{gathered}
$$

$$
\begin{aligned}
& G_{n} \varphi\left(x_{1}, \cdots \cdots, x_{n}\right)=\sum_{N=0}^{n-1} Q_{n}(N) \varphi\left(x_{1}, \cdots \cdots, x_{n}\right), \\
& G_{n} \varphi_{1} G_{n} \varphi_{2} \cdots \cdots \cdot G_{n} \varphi_{k}=\sum_{N_{k}=0}^{n-1} \cdots \cdots \cdot \sum_{N_{1}=0}^{n-1} Q_{n}\left(N_{k}\right) \varphi_{k} \cdots \cdots Q_{n}\left(N_{1}\right) \varphi_{1} .
\end{aligned}
$$

Here, we mean, for instance, $G_{n} \varphi_{2} G_{n} \varphi_{1}=G_{n}\left(\varphi_{2} \cdot G_{n} \varphi_{1}\right)$

LEMMA 6. If $\varphi_{1}, \cdots \cdots, \varphi_{k}$ have indices in $I_{0}=\{1,2, \cdots \cdots, M\}$ and $n>M$, then,

$$
Q_{n}\left(N_{k}\right) \varphi_{k} \cdots \cdots Q_{n}\left(N_{1}\right) \varphi_{1} \equiv \frac{(n-M) !}{\left(n-M-N_{1}-\cdots \cdots-N_{k}\right) ! n^{N_{1}+\cdots+N_{k}}} Q\left(N_{k}\right) \varphi_{k} \cdots \cdots
$$


where

$$
\left.\cdots \cdots Q\left(N_{1}\right) \varphi_{1}+e_{n}\left(N_{k}, \cdots \cdots, N_{1} ; \varphi_{k}, \cdots \cdots, \varphi_{1}\right) \quad\left(\bmod I_{0}^{c}\right), *\right)
$$

$$
\begin{aligned}
& \sum_{N_{k}=0}^{n-1} \cdots \cdots \sum_{N_{1}=0}^{n-1}\left\|e_{n}\left(N_{k}, \cdots \cdots, N_{1} ; \varphi_{k}, \cdots \cdots, \varphi_{1}\right)\right\| \\
& \quad \leq \frac{2}{n}(k+2) !\left(2 L\left(2+C_{0}\right)\right)^{k+2} e^{M / L}\left\|\varphi_{1}\right\| \cdots \cdots\left\|\varphi_{k}\right\|
\end{aligned}
$$

for any possible choice of indices for $Q\left(N_{k}\right), \cdots \cdots, Q\left(N_{1}\right)$.

Proof. We have, by definition,

$$
\begin{aligned}
& Q_{n}\left(N_{k}\right) \varphi_{k} \cdots \cdots Q_{n}\left(N_{1}\right) \varphi_{1} \\
& =n^{-\left(N_{1}+\ldots+N_{k}\right)} \quad \Sigma^{(n)} \ldots \ldots \Sigma^{(n)} \quad \sum^{(n)} \\
& \left(j_{1}^{k}, \ldots, j_{N_{k}}^{k}, i_{k}\right)\left(j_{1}^{2}, \ldots, j_{N_{2}}, i_{2}\right)\left(j_{1}, \ldots,{ }_{N_{1}}, i_{1}\right) \\
& \left.A_{N_{k}}^{\left(i_{k}\right)}\left(x_{j k}, \cdots \cdots, x_{N_{k} k}\right) \varphi_{k} \cdot A_{N_{k-1}}^{\left(i_{k-1}\right)} \underset{1}{x_{j k-1}}, \cdots \cdots, x_{N_{k-1}}\right) \varphi_{k-1} \cdots \cdots \varphi_{2} \cdot A_{N_{1}}^{\left(i_{1}\right)} \\
& \left(x_{j 1}, \cdots \cdots, x_{j 1} N_{1}\right) \varphi_{1},
\end{aligned}
$$

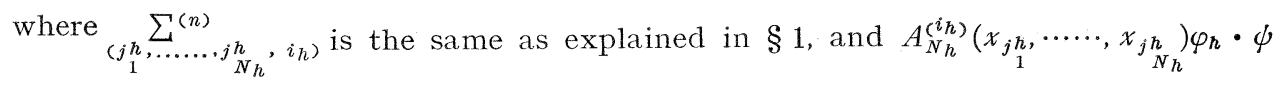
means the following function:

$$
\int_{S} A N_{h}\left(x_{j h}, \cdots \cdots, x_{j h} \mid x_{i_{h}}, d y\right)\left(\varphi_{h} \cdot \phi\right)(x_{1}, \cdots, \overbrace{y}^{i_{h}}, \cdots, x_{n}) .
$$

Now, the number of such $J=\left(j_{1}^{1}, \cdots \cdots, j_{N_{1}}^{1}, \cdots, j_{1}^{k}, \cdots \cdots, j_{N_{k}}^{k}\right)$, where no number appears more than once and each $j_{i}^{h}$ belongs to $\{M+1, \cdots \cdots, n\}$, is equal to

$$
(n-M)(n-M-1) \cdots\left(n-M-N_{1}-\cdots \cdots-N_{k}+1\right)=(n-M) ! /\left(n-M-N_{1}-\cdots \cdots-N_{k}\right) !
$$
and the sum over $\left(i_{1}, \cdots \cdots, i_{k}\right)$ of those terms with one fixed $J$ of this sort is equivalent modulo $I_{0}^{c}$ to

$$
n^{-\left(N_{1}+\ldots+N_{k}\right)} Q\left(N_{k}\right) \varphi_{k} \cdots \cdots Q\left(N_{1}\right) \varphi_{1} \cdot
$$

Hence, the sum of all terms with such $J$ is the first summand of (4.2).

On the other hand, the number of $J^{\prime} s$ where at least one of $j_{i}^{h}$ belongs to $\{1$, , $M\}$ is bounded by

$$
\left(N_{1}+\cdots \cdots+N_{k}\right) M n^{N_{1}+\ldots+N_{k}-1}
$$

The number of $J$, s, where some $j_{i}^{h}$ and $j_{i}^{h^{\prime}}$ coincide for some $h \neq h^{\prime}$ is bounded by

$$
\begin{gathered}
{\left[N_{1}\left(N_{2}+\cdots \cdots+N_{k}\right)+N_{2}\left(N_{3}+\cdots \cdots+N_{k}\right)+\cdots \cdots+N_{k-1} N_{k}\right] n^{N_{1}+\ldots N_{k}-1}} \\
\leq\left(N_{1}+\cdots \cdots+N_{k}\right)^{2} \cdot n^{N_{1}+\ldots+N_{k}-1},
\end{gathered}
$$

since the number of $J^{\prime} s$, where some $j_{i}^{h}$ coincides with some $j_{i^{\prime}}^{h^{\prime}}$ for $h^{\prime}>h$, but not for $h^{\prime}<h$, is bounded by $N_{h}\left(N_{h+1}+\cdots \cdots+N_{k}\right) n^{N_{1}+\ldots+N_{k}-1}$. And each partial sum over $\left(i_{1}, \cdots, i_{k}\right)$ with one fixed $J$ is bounded by

$$
n^{-\left(N_{1}+\ldots+N_{k}\right)} M\left(M+N_{1}\right) \cdots\left(M+N_{1}+\cdots \cdots+N_{k-1}\right) C_{N_{1}} \cdots \cdots C_{N_{k}}\left\|\varphi_{1}\right\| \cdots \cdots\left\|\varphi_{k}\right\| \text {. }
$$

*) By the first term of the right hand side of (4.2) we mean 0 when $N_{1}+\cdots \cdots+N_{k}>n-M$. 
Thus, the partial sum $e_{n}\left(N_{k}, \cdots \cdots, N_{1} ; \varphi_{k}, \cdots \cdots, \varphi_{1}\right)$ is bounded by

$$
\begin{aligned}
& \left\{\frac{1}{n} M\left(N_{1}+\cdots \cdots+N_{k}\right)+\frac{1}{n}\left(N_{1}+\cdots \cdots+N_{k}\right)^{2} M\left(M+N_{1}\right) \cdots \cdots\left(M+N_{1}+\cdots \cdots+N_{k-1}\right)\right\} \\
& C_{N_{1}} \cdots \cdots C_{N_{k}}\left\|\varphi_{1}\right\| \cdots \cdots\left\|\varphi_{k}\right\| \leq \frac{2}{n}\left(N_{1}+\cdots \cdots+N_{k}+M\right)^{k+2} C_{N_{1}} \cdots \cdots C_{N_{k}}\left\|\varphi_{1}\right\| \cdots \cdots\left\|\varphi_{k}\right\|
\end{aligned}
$$

Then, by a formula of Dudley Johnson $[2, p .283]$ :

$$
\sum_{N_{1}^{1}=1}^{\infty} \cdots \cdots \sum_{N_{k}=1}^{\infty}\left(M+N_{1}+\cdots \cdots+N_{k}\right)^{q} C_{N_{1}} \cdots \cdots C_{N_{k}} \leq q !(2 L)^{q} e^{M / L} \cdot 2^{k},
$$

we have the following estimate:

$$
\begin{aligned}
\sum_{N_{k}=0}^{n-1} \cdots \cdots & \sum_{N_{1}=0}^{n-1}\left\|e_{n}\left(N_{k}, \cdots \cdots, N_{1} ; \varphi_{k} \cdots \cdots \varphi_{1}\right)\right\| \\
& \leq \frac{2}{n}\left\|\varphi_{1}\right\| \cdots \cdots\left\|\varphi_{k}\right\| \sum_{N_{1}=0}^{\infty} \cdots \cdots \sum_{N_{k}=0}^{\infty}\left(N_{1}+\cdots \cdots+N_{k}+M\right)^{k+2} C_{N_{1}} \cdots \cdots C_{N_{k}} \\
& \leq \frac{2}{n}\left\|\varphi_{1}\right\| \cdots \cdots\left\|\varphi_{k}\right\| \sum_{h=0}^{k}\left(\begin{array}{l}
k \\
h
\end{array}\right) C_{0}^{h} \sum_{N_{h}+1}^{\infty} \cdots \cdots \sum_{N_{k}=1}^{\infty}\left(N_{h+1}+\cdots \cdots+N_{k}+M\right)^{k+2} C_{N_{h}+1} \cdots \cdots C_{N_{1}} \\
& \leq \frac{2}{n}\left\|\varphi_{1}\right\| \cdots \cdots\left\|\varphi_{k}\right\|(k+2) !(2 L)^{k+2} e^{M / L} \sum_{h=0}^{k}\left(\begin{array}{l}
k \\
h
\end{array}\right) C_{0}^{h} 2^{k-h} \\
& \leq \frac{2}{n}(k+2) !\left(2 L\left(2+C_{0}\right)\right)^{k+2} e^{M / L}\left\|\varphi_{1}\right\| \cdots \cdots \cdot \varphi_{k} \| .
\end{aligned}
$$

THEOREM 4. If $\varphi_{1}, \cdots \cdots, \varphi_{k}$ have indices in $I_{0}=\{1, \cdots \cdots, M\}$, and $t_{1}+\cdots \cdots+t_{k}$ $<1 / 2 L\left(M+C_{0}+1\right)$, then,

$$
\begin{aligned}
e^{t_{k} G_{n}} \varphi_{k} \cdots \cdots e^{t_{1} G_{n}} \varphi_{1}-e^{t_{k} D} \varphi_{k} \cdots \cdots e^{t_{1} D} \varphi_{1} & \\
& \equiv e_{n}\left(t_{1}, \cdots \cdots, t_{k} ; \varphi_{1}, \cdots \cdots, \varphi_{k}\right) \quad\left(\bmod I_{0}{ }^{c}\right),
\end{aligned}
$$

where

$$
\left\|e_{n}\left(t_{1}, \cdots, t_{k} ; \varphi_{1}, \cdots \cdots, \varphi_{k}\right)\right\| \rightarrow 0 \text {, as } n \rightarrow \infty \text {, }
$$

for any possible choice of indices.

Proof. We have, by Lemma 6,

$$
\begin{aligned}
& e^{t_{k} G_{\bar{n}}} \varphi_{k} \cdots \cdots e^{t_{1} G_{n}} \varphi_{1}-e^{t_{k} D} \varphi_{k} \cdots \cdots e^{t_{1} D} \varphi_{1} \\
& =\sum_{p_{1}=0}^{\infty} \cdots \cdots \sum_{p_{k}=0}^{\infty} \frac{t_{1} p_{1} \cdots \cdots t_{k} p_{k}}{p_{1} ! \cdots \cdots p_{k} !}\left(G_{n}^{p_{k}} \varphi_{k} \cdots \cdots G_{n}^{p_{1}} \varphi_{1}-D^{p_{k}} \varphi_{k} \cdots \cdots D^{p_{1}} \varphi_{1}\right) \\
& =\sum_{p_{1}=0}^{\infty} \cdots \cdots \sum_{p_{k}=0}^{\infty} \frac{t_{1} p_{1} \cdots \cdots t_{k} p_{k}}{p ! \cdots \cdots p_{k} !}\left(\sum_{N_{p_{1}}+\cdots+p_{k}=0}^{n-1} \cdots \cdots \cdots \sum_{N_{1}=0}^{n-1} Q_{n}\left(N_{p_{1}}+\cdots \cdots+p_{k}\right) \cdots \cdots\right. \\
& Q_{n}\left(N_{p_{1+} \cdots+p_{k-1}+1}\right) \varphi_{k} \cdots \cdots \varphi_{2} Q_{n}\left(N p_{1}\right) \cdots \cdots Q_{n}\left(N_{1}\right) \varphi_{1} \\
& -\sum_{p_{1}+\cdots+p_{k}=0}^{\infty} \cdots \sum_{N 1=0}^{\infty} Q\left(N p_{1}+\cdots+p_{k}\right) \cdots \cdots Q\left(N_{p_{1}+\cdots+p_{k-1}+1}\right) \varphi_{k} \cdots \cdots \varphi_{2} Q\left(N_{p_{1}}\right) \cdots \cdots Q\left(N_{1}\right) \varphi_{1} \\
& \equiv \sum_{p_{1}=0}^{\infty} \cdots \cdots \sum_{p_{k}=0}^{\infty} \frac{t_{1}^{p_{1} \cdots \cdots \cdot t_{k} p_{k}}}{p_{1} ! \cdots \cdots p_{k} !} \sum_{N p_{1}+\cdots+p_{k}=0}^{n-1} \ldots \ldots \sum_{N_{1}=0}^{n-1} \\
& \left(\frac{(n-M) !}{\left(n-M-N_{1}-\cdots \cdots-N p_{1}+\cdots+p_{k}\right) ! n^{N_{1}+\cdots+N p_{1}+\cdots+p_{k}}}-1\right) \\
& Q\left(N_{p_{1}+\cdots+p_{k}}\right) \cdots \cdots Q\left(N_{p_{1}+\cdots+p_{k-1}+1}\right) \varphi_{k} \cdots \cdots \varphi_{2} Q\left(N_{p_{1}}\right) \cdots \cdots Q\left(N_{1}\right) \varphi_{1}
\end{aligned}
$$




$$
\begin{aligned}
& +\sum_{p_{1}=0}^{\infty} \cdots \cdots \sum_{p_{k}=0}^{\infty} \frac{t_{1} p_{1} \cdots \cdots t_{k} p_{k}}{p_{1} ! \cdots \cdots p_{k} !} \sum_{N p_{1}+\cdots+p_{k}=0}^{n-1} \cdots \cdots \sum_{N_{1}=0}^{n-1} e_{n}\left(N_{p_{1}+\cdots+p_{k}}, \cdots \cdots, N_{2}, N_{1} ;\right. \\
& -\sum_{p_{1}=0}^{1, \cdots \cdots, 1}, \varphi_{k}, 1, \cdots \cdots, \varphi_{2}, \underbrace{1, \cdots \cdots, 1}_{p_{k}=0}, \varphi_{1}) \\
& =I_{1}+I_{2}+I_{3}\left(\bmod I_{0}{ }^{c}\right),
\end{aligned}
$$

Here, $\quad \sum_{\left(N_{1}<n, \ldots, N p_{1}+\cdots+p_{k}<n\right) e}$ means the sum over $\left(N_{1}, \cdots \cdots, N_{p_{1}+\cdots+p_{k}}\right)$, where at least one of $N_{i}$ is larger than $n\left(1 \leq i \leq p_{1}+\cdots \cdots+p_{k}\right)$.

By Lemma 6, $\left\|I_{2}\right\|$ is bounded by

$$
\text { (4.3) } \begin{gathered}
\sum_{p_{1}=0}^{\infty} \cdots \cdots \sum_{p_{k}=0}^{\infty} \frac{t_{1} p_{1} \cdots \cdots t_{k} p_{k}}{p_{1} ! \cdots \cdots p_{k} !}\left(p_{1}+\cdots \cdots+p_{k}+2\right) !\left(2 L\left(2+C_{0}\right)\right)^{p_{1}+\cdots+p_{k}+2} \frac{2}{n} e^{M / L}\left\|\varphi_{1}\right\| \cdots\left\|\varphi_{1}\right\| \\
=\frac{2}{n} e^{M / L} 2\left(1-2 L\left(2+C_{0}\right)\left(t_{1}+\cdots \cdots+t_{k}\right)\right)^{-3}\left(2 L\left(2+C_{0}\right)\right)^{3}\left\|\varphi_{1}\right\| \cdots \cdots\left\|\varphi_{k}\right\|,
\end{gathered}
$$

and hence, $\left\|I_{2}\right\|$ is arbitrarily small for sufficiently large $n$. As we have seen in the proof of Lemma 2, we have

$$
\begin{gathered}
\sum_{p_{1}=0}^{\infty} \cdots \cdots \sum_{p_{k}=0}^{\infty} \frac{t_{1} p_{1} \cdots \cdots t_{k} p_{k}}{p_{1} ! \cdots \cdots p_{k} !} \sum_{N_{1}=0}^{\infty} \sum_{N_{2}=0}^{\infty} \cdots \cdots \sum_{N p_{1}+\cdots+p_{k}=0}^{\infty} \| Q\left(N_{p_{1}+\cdots+p_{k}}\right) \cdots \cdots \\
\cdots \cdots Q\left(N_{p_{1}+\cdots+p_{k-1}+1}\right) \varphi_{k} \cdots \cdots Q\left(N_{2}\right) Q\left(N_{1}\right) \varphi_{1} \|<\infty,
\end{gathered}
$$

and hence, $\left\|I_{3}\right\|$ is also arbitrarily small for sufficiently large $n$. Finally, $I_{1}$ is wirtten as

where

$$
\begin{aligned}
\sum_{p_{1}=0}^{\infty} \cdots \cdots \sum_{p_{k}=0}^{\infty} \frac{t_{1} p_{1} \cdots \cdots t_{k} p_{k}}{p_{1} ! \cdots \cdots p_{k} !} \sum_{N p_{1}+\cdots+p_{k}=0}^{\infty} \cdots \sum_{N_{1}=0}^{\infty} a\left(n, M, N_{1}, \cdots \cdots, N p_{1}+\cdots+p_{k}\right) \\
Q\left(N_{p_{1}+\cdots+p_{k}}\right) \cdots \cdots Q\left(N p_{1}+\cdots+p_{k-1}+1\right) \varphi_{k} \cdots \cdots \varphi_{2} Q\left(N p_{1}\right) \cdots \cdots Q\left(N_{1}\right) \varphi_{1},
\end{aligned}
$$

$$
\begin{aligned}
a\left(n, M, N_{1}, \cdots \cdots, N_{p_{1}+\cdots+p_{k}}\right) & =\frac{(n-M) !}{\left(n-M-N_{1}-\cdots \cdots-N p_{1+\cdots+p_{k}}\right) ! n^{N_{1}+\cdots+N p_{1}+\cdots+p}}-1, \\
& =0, \text { if otherwise. } 0 \leq N_{1}, \cdots \cdots, N p_{1+\cdots+p_{k}} \leq n-1,
\end{aligned}
$$

Then, by the bounded convergence theorem, (4.4) and

$$
\begin{aligned}
& -1 \leq a\left(n, M, N_{1}, \cdots \cdots, N p_{1}+\cdots+p_{k}\right) \leq 0 \\
& a\left(n, M, N_{1}, \cdots \cdots, N p_{1}+\cdots+p_{k}\right) \rightarrow 0, \text { as } n \rightarrow \infty
\end{aligned}
$$

imply that $I_{1} \rightarrow 0$, as $n \rightarrow \infty$.

COROLlaRy. $\quad P_{J}^{f}\left(t, x_{1}, \cdots \cdots, x_{h} ; \cdot\right)$ and $f_{t, J}(\cdot)$ of Lemma 3 are probability measures on $\left(S^{J}, \boldsymbol{B}\left(S^{J}\right)\right)$, where $J=\{1,2, \cdots \cdots, k\}, h \leq k$, and $t<1 / 2\left[2 L\left(k+C_{0}+1\right)\right]$.

Proof. Let $J_{1}=\{1,2, \cdots \cdots, h\}$ and take $I_{0}$ so that $J \subset I_{0}$. Since $\phi_{1} \equiv \phi_{2}\left(\bmod I_{0}{ }^{c}\right)$ implies $\int_{J_{1}^{c}} f^{\infty} \phi_{1}=\int_{J_{1}^{c}} f^{\infty} \phi_{2}$, we have, by Theorem 4,

$$
\int_{J_{1}^{c}} f^{\infty} e^{t D} \varphi=\lim _{n \rightarrow \infty} \int_{J^{c}} f^{\infty} e^{t G n} \varphi
$$


for $t<1 /\left[2 L\left(k+C_{0}+1\right)\right]$ and $\varphi \in F_{0}$ with $I(\varphi)=J$. But, the left hand side is $\int_{S J} P_{J}^{f}\left(t, x, \cdots \cdots, x_{h} ; d y_{1} \cdots \cdots d y_{k}\right) \varphi\left(y_{1}, \cdots \cdots, y_{k}\right)$, while the limitand on the right is non-negative, if $\varphi$ is non-negative. The right hand side is clearly 1 , if $\varphi \equiv 1$. Thus, $P_{J}^{f}\left(t, x_{1}, \cdots \cdots, x_{h} ; \cdot\right)$ is a probability measure on $S^{J}$, implying that $f_{t, J}$ is also a probability measure by $(3.4)$.

Proof of Theorem 2. Let $\varphi_{h}\left(x_{1}\right)=\chi_{A_{h}}\left(x_{1}\right), \phi_{h}\left(x_{2}\right)=\chi_{B_{h}}\left(x_{2}\right), h=1,2, \cdots \cdots, k$, and let $I_{0}=\{1,2\}$, where $\chi_{A}(x)$ is defined by $\chi_{A}(x)=1$ or 0 , according as $x \in A$ or not. Now, the left hand side of (1.10) is equal to

$$
\begin{aligned}
& \lim _{n \rightarrow \infty} \int_{S n} f^{(n)} e^{t_{1} G_{n}} \varphi_{1} \psi_{1} e^{\left(t_{2}-t_{1}\right) G_{n}} \varphi_{2} \psi_{2} \cdots \cdots e^{\left(t_{k}-t_{k-1}\right) G_{n}} \varphi_{k} \psi_{k} \\
& \quad=\lim _{n \rightarrow \infty} \int f^{\infty} e^{t_{1} G_{n}} \varphi_{1} \psi_{1} e^{\left(t_{2}-t_{1}\right) G_{n}} \varphi_{2} \phi_{2} \cdots \cdots e^{\left(t_{k}-t_{k}-1\right) G_{n}} \varphi_{k} \phi_{k}
\end{aligned}
$$

which is, by Theorem 4 , equal to

But, by (2.9) in the Corollary to Lemma 1 , this is equal to

$$
\int f^{\infty} e^{t_{1} D} \varphi_{1} \phi_{1} \cdots \cdots e^{\left(t_{k}-t_{k-1}\right) D} \varphi_{k} \psi_{k}, \quad \text { for } t_{k}<\frac{1}{2 L\left(3+C_{0}\right)} .
$$

$$
\begin{gathered}
\int f^{\infty}\left(e^{t_{1} D} \varphi_{1} e^{\left(t_{2}-t_{1}\right) D} \varphi_{2} \cdots \cdots e^{\left(t_{k}-t_{k-1}\right) D} \varphi_{k}\right) \otimes\left(e^{t_{1} D} \phi_{1} \cdots \cdots e^{\left(t_{k}-t_{k-1}\right) D} \phi_{k}\right) \\
=\int f^{\infty} e^{t_{1} D} \varphi_{1} \cdots \cdots e^{\left(t_{k}-t_{k-1}\right) D} \varphi_{k} \cdot \int f^{\infty} e^{t_{1} D} \phi_{1} \cdots \cdots e^{t_{k} D} \phi_{k} \\
=P^{f}\left(x\left(t_{1}\right) \in A_{1} \cdots \cdots x\left(t_{k}\right) \in A_{k}\right) \cdot P^{f}\left(x\left(t_{1}\right) \in B_{1} \cdots \cdots x\left(t_{k}\right) \in B_{k}\right),
\end{gathered}
$$

by Theorem 3. The second equality of $(1.10)$ is now clear.

\section{$\S 5$. Examples and comments}

a) Consider an equation

(5.1) $\quad \frac{\partial u(t, x)}{\partial t}=\frac{1}{2 \pi} \int_{R^{1} \times[0,2 \pi)}\left[u\left(t, x^{*}\right) u\left(t, y^{*}\right)-u(t, x) u(t, y)\right] d y d \theta$

where

$$
x^{*}=x \cos \theta-y \sin \theta, y^{*}=x \sin \theta+y \cos \theta
$$

and $u(t, x)$ is supposed to be a density function of a probability measure on $S=R^{1}$ with respect to the Lebesgue measure. Clearly, (5.1) corresponds to the one dimensional model of Maxwellian gas given by Mark Kac [3].*) Since this is equivalent to

$$
\frac{\partial u(t, x)}{\partial t}=\frac{1}{\pi} \iint_{x * 2+y * 2>x^{2}} \frac{u\left(t, x^{*}\right) u\left(t, y^{*}\right)}{\sqrt{x^{* 2}+y^{* 2}-x^{2}}} d x^{*} d y^{*}-u(t, x),
$$

we can rewrite (5.1) in the following way.

$$
\frac{\partial u(t, \cdot)}{\partial t}=\int_{R^{2}} u(t, d x) u\left(t, d x_{1}\right) A_{1}\left(x_{1} \mid x, \cdot\right)
$$

where

$$
A_{1}\left(x_{1} \mid x, E\right)=\frac{1}{\pi} \int_{E \cap\left(y \mid x^{2}+x_{1}^{2}>y^{2}\right)} \frac{d y}{\sqrt{x^{2}+x_{1}^{2}-y^{2}}}-\delta_{x}(E) .
$$

This is just (1.2), where

$$
A_{0}(x, \cdot) \equiv A_{2}\left(x_{1}, x_{2} \mid x, \cdot\right) \equiv \cdots \cdots \equiv 0,
$$

*) See also McKean $[5,6]$. 


$$
q_{1}\left(x_{1} \mid x\right) \equiv 1, \quad \pi_{1}\left(x_{1} \mid x, E\right)=\frac{1}{\pi} \int_{E \cap\left(x^{2}+x_{1}^{2}>y^{2}\right)} \frac{d y}{\sqrt{x^{2}+x_{1}^{2}-y^{2}}} .
$$

This corresponds to the situation that the motion is determined by interactions between two particles...... binary collisions alone. Here, the hitting measure depends on the distribution $u(t, \cdot)$ at time $t$, but the waiting time for jumps is independent on $u(t, \cdot)$.

b) In case $S=\{1,-1\}$, the mass of $\pi_{N}$ necessarily concentrates at one point, and hence,

$$
\pi_{N}\left(x, \cdots \cdots, x_{N} \mid x, \cdot\right)=\delta_{\{-x\}}(\cdot), x= \pm 1,
$$

independently on $N$ and $x_{1}, \cdots \cdots, x_{N} . q_{N}$ being symmetric in $x_{1}, \cdots, \ldots, x_{N}$, there is a non-negative function $C_{N}(x, k)$ in $(x, k) \in S \times\{1, \cdots \cdots, N\}$ such that

$q_{N}\left(x_{1}, \cdots \cdots, x_{N} \mid x\right)=C_{N}\left(x\right.$, the number of $1^{\prime} \mathrm{s}$ in $\left.\left\{x_{1}, \cdots \cdots, x_{N}\right\}\right)$, where $C_{N}(x, \cdot)$ satisfies the growth condition.

$$
\sum_{N=0}^{\infty} N^{P}\left(\max _{x, k} C_{N}(x, k)\right)=\frac{1}{2} \sum_{N=0}^{\infty} N^{P}\left\|A_{N}\right\| \leq \frac{1}{2} L^{P} \cdot P \text { ! }
$$

Then, $A^{(u)}(x, \cdot)$ is given, writing $u(\{1\})=p$ and $u(\{-1\})=q, 1-p$ by

$$
\begin{aligned}
A^{(u)}(x, \cdot) & =\sum_{N=0}^{\infty} \int_{S N} \prod_{i=1}^{N} u\left(d x_{i}\right) q_{N}\left(x_{1}, \cdots \cdots, x_{N} \mid x\right)\left(\delta_{\{x\}}(\cdot)-\delta_{\{x\}}(\cdot)\right) \\
& =\sum_{N=0}^{\infty} \sum_{k=0}^{N}\left(\begin{array}{l}
N \\
k
\end{array}\right) p^{k}(1-p)^{N-k} C_{N}(x, k)\left(\delta_{\{-x\}}(\cdot)-\delta_{\{x\}}(\cdot)\right) \\
& =a(x, p)\left(\delta_{\{-x\}}(\cdot)-\delta_{\{x\}}(\cdot)\right) .
\end{aligned}
$$

Hence, equation (1.1) can be rewritten as

or, equivalently,

$$
\begin{aligned}
\frac{d}{d t}\left(p_{t}, q_{t}\right)=\left(p_{t}, q_{t}\right) & \left(\begin{array}{l}
-a\left(1, p_{t}\right), \\
a\left(-1, p_{t}\right),-a\left(1, p_{t}\right)
\end{array}\right) \\
\left(p_{t}, q_{t}\right) & \rightarrow\left(p_{0}, q_{0}\right),
\end{aligned}
$$

$$
\begin{gathered}
\frac{d}{d t} p_{t}=-a\left(1, p_{t}\right) p_{t}+a\left(-1, p_{t}\right)\left(1-p_{t}\right), \\
p_{t} \rightarrow p_{0} .
\end{gathered}
$$

This is the case which Dudley Johnson described in $[1,2]$. He characterized the class of functions $a(x, p)$ of $p$, expanded as in (5.4) with non-negative $C_{N}(x, k)$ satisfying the growth condition (5.3), as the set of all functions which are nonnegative, analytic on $[0,1]$ and positive on $(0,1)$. We easily obtain a similar result, when $S=\{1,2, \cdots \cdots, m\}$ and

$$
\begin{aligned}
A^{(u)}(x, \cdot) & =\sum_{N=0}^{\infty} \sum_{k_{1}+\cdots+k_{m}=N}\left(\begin{array}{c}
N \\
k_{1}, \cdots \cdots, k_{m}
\end{array}\right) p_{1}^{k_{1}} \cdots \cdots \cdot p_{m}^{k_{m}} C_{N}\left(k_{1}, \cdots \cdots, k_{m} \mid x\right) \cdot\left(\pi(x, \cdot)-\delta_{\{x\}}(\cdot)\right) \\
& =a\left(x, p_{1}, \cdots \cdots, p_{m}\right)\left(\pi(x, \cdot)-\delta_{x}(\cdot)\right),
\end{aligned}
$$

where $C_{N}$ satisfies $\sum_{N=0}^{\infty} N^{p}\left(\max C_{N}\right) \leq \frac{1}{2} L^{p} \cdot p !$, and $\pi(x, \cdot)$ is a probability measure on $S$ with $\pi(x,\{x\})=0$. It is easy to prove that $a\left(x, p_{1}, \cdots \cdots, p_{m}\right)=a\left(x, p_{1}, \cdots \cdots, p_{m_{-1}}\right.$, $\left.1-\sum_{1}^{m-1} p_{k}\right)$ is non-negative, analytic in $p_{1}, \cdots \cdots, p_{m_{-1}}$ on $\left\{\left(p_{1}, \cdots \cdots, p_{m-1}\right) \mid 0 \leq p_{1}, \cdots \cdots\right.$, $\left.p_{m-1}, p_{1}+\cdots \cdots+p_{m-1} \leq 1\right\}$, and positive at inner points, while the converse is still open for $m>2$. 
c) Let $S=\{\cdots \cdots,-1,0,1,2, \cdots \cdots\}$ and consider

$$
\begin{gathered}
\frac{\partial}{\partial t} u(t, x)=\frac{1}{2}\left(u(t, x+1)^{N+1}+u(t, x-1)^{N+1}-2 u(t, x)^{N+1}\right), \\
u(t, \cdot) \rightarrow f(\cdot), t \rightarrow 0 .
\end{gathered}
$$

This is a discrete model of the non-linear equation

$$
\frac{\partial}{\partial t} u(t, x)=\Delta\left(u(t, x)^{N+1}\right), \quad u(t, x) \rightarrow f(x), t \rightarrow 0
$$

and can be discussed in our scheme, by taking

$$
\begin{array}{r}
A_{0} \equiv A_{1} \equiv \cdots \cdots \equiv A_{N-1} \equiv A_{N+1} \equiv \cdots \cdots \equiv 0 \\
q_{N}\left(x_{1}, \cdots \cdots, x_{N} \mid x\right)=1, \text { if } x_{1}=\cdots \cdots=x_{N}=x \\
=0, \text { if otherwise, } \\
\pi_{N}\left(x_{1}, \cdots \cdots, x_{N} \mid x, \cdot\right)=\frac{1}{2}\left(\delta_{\{x+1\}}(\cdot)+\delta_{\{x-1\}}(\cdot)\right) .
\end{array}
$$

Similarly, a discrete model of the following equation can be considered,

$$
\frac{\partial}{\partial t} u(t, x)=\frac{\partial^{2}}{\partial x^{2}}(F(u(t, x)))+\frac{\partial}{\partial x}(G(u(t, x)))
$$

where $F$ and $G$ (or $-G$ ) are polynomials or analytic functions satisfying a suitable growth condition with positive coefficients. For instance, a discrete model of Burger's equation*) is given by

$$
\frac{\partial}{\partial t} u(x)=\frac{1}{2}(u(x+1)+u(x-1)-2 u(x))-\frac{1}{2}\left(u(x+1)^{2}-u(x-1)^{2}\right) .
$$

d) As far as the equation (1.1) is concerned, an arbitrary signed measure or a complex valued measure $f$ with bounded variation can be an initial value, in which case the solution $u(t, x)$ is a signed measure or a complex valued measure. In fact, (2.5) shows that we do not really need an infinite product measure $f^{\infty}$ when we integrate a function in $F$. Only the time interval, in which expansions. of exponentials have meaning, needs to be changed by simply multiplying $1 /\|f\|$.

Moreover, $A^{(u)}(x, \cdot)$ need not be a stochastic generator kernel for the equation. In fact, $A_{N}\left(x_{1}, \cdots \cdots, x_{N} \mid x, \cdot\right)$ in $\S 1$ can be replaced by an arbitrary complex valued kernel satisfying the measurability in $\left(x_{1}, \cdots \cdots, x_{N}, x\right)$, symmetry in $\left(x_{1}, \cdots \cdots, x_{N}\right)$ and the condition (1.3) on the total variation. The same is true also for a linear combination of such kernels. Theorem 4 also remains true for such kernels.

\section{References}

[1] D. P. Johnson, thesis, Massachusetts Institute of Technology (unpublished).

[2] - On a class of stochastic processes and its relationship to infinite particle gases, Trans. Amer. Math. Soc., 132 (1968), 275-295.

[3] M. Kac, Probability and related topics in the physical sciences, Wiley, New York, 1959.

[4] H. P. McKean, Jr., A class of Markov processes associated with non-linear parabolic equations, Proc. Nat. Acad. Sci., 56 (1966), 1907-1911.

[5] - Speed of approach to equilibrium for Kac's caricature of a Maxwellian gas, Archive Rat. Mech. Anal., 21 (1966), 343-367.

[6] - An exponential formula for solving Boltzmann's equation for a Maxwellian gas, Journal of Combinatorial Theory, 2 (1967), 358-382.

THE UNIVERSITY OF TOKYO and THE ROCKEFELLER UNIVERSITY

*) See McKean $[4,6]$. 